\title{
Kollokationen - ein vernachlässigtes Gebiet der DaF-Didaktik?
}

\author{
Joanna Targońska (Olsztyn)
}

\begin{abstract}
Collocations, a highly specific group of set phrases, play an important role in the process of foreign language learning and acquisition. While their importance is unquestioned, they constitute a frequent source of errors, because the learners do not focus their attention on a collocation's form. What's more, they frequently assume that specific fixed sequences of words may be freely transferred from one language to another. In this paper we argue that collocations are in fact a neglected or even unrecognized aspect of teaching German as a foreign language. First, we discuss the relevance of the issue in foreign language teaching and learning. Next, we present an overview of research on collocations. Finally, we turn to an account of the results of our empirical study supporting the thesis as framed in the title.
\end{abstract}

\section{Einleitung}

Kollokation ist ein Begriff, der in der englischsprachigen Fremdsprachen(erwerbs)forschung sowie in der EaF-Didaktik sehr oft gebraucht wird, deren Bedeutung jedoch in der DaF-/DaZDidaktik nicht ganz erkannt worden zu sein scheint. Schon vor über 15 Jahren stellte Barrios (1997) fest, dass Kollokationen in der Wortschatzarbeit im DaF-Unterricht vernachlässigt werden. ${ }^{1}$ Man könnte sich nach 17 Jahren fragen, ob Kollokationen inzwischen als ein wichtiges sprachliches Phänomen erkannt und betrachtet werden. Zwar muss als positiv bewertet werden, dass sich schon seit Anfang der 80er Jahre die Phraseodidaktik in der DaF-/DaZ-Forschung zu etablieren begann, ${ }^{2}$ aber diese konzentriert sich in erster Linie auf Phraseologismen im engeren Sinne (also auf Wortidiome bzw. Phraseolexeme) sowie in viel geringerem Unfang auf Routineformeln bzw. kommunikative Formeln, komparative Phraseologismen, Sprichwörter bzw. sprichwörtliche Redensarten, wobei Kollokationen als eine spezifische Art der Phraseme immerhin als eine Randerscheinung betrachtet zu werden scheinen. ${ }^{3}$ Auch die Tatsache, dass eine Nummer der Zeitschrift Linguistik online der Phraseodidaktik gewidmet wurde

\footnotetext{
${ }^{1}$ In seinem Artikel unter dem Titel: "Kollokationen - ein vernachlässigtes Thema in der Wortschatzarbeit" konzentriert sich Barrios (1997) nicht auf die Bestätigung der im Titel formulierten These. Obwohl der Titel des vorliegenden Artikels an den Titel von Barrios' Beitrag anknüpft, wird dieses auf die Überprüfung der im Titel formulierten These ausgerichtet.

2 Ettinger (1998: 204) verweist darauf, dass schon Ende der 70er Jahre Glaap (1979) und Weller (1979) sich mit der Phraseodidaktik befassten, wobei der Begriff Phraseodidaktik in Deutschland noch nicht explizit gebraucht wurde und ihre didaktischen Überlegungen noch nicht im Rahmen der Phraseodidaktik geäußert wurden. Diese können jedoch als Pionierarbeiten der deutschen Phraseodidaktik gedeutet werden.

3 An dieser Stelle ist darauf hinzuweisen, dass Jahrzehnte lang Phraseologismen eine viel engere Gruppe spezifischer Wortverbindungen bildeten, zu der Phraseologismen und Idiome jedoch keine Kollokationen gehörten, was Hausmann (2007: 217) folgendermaßen beschrieb: "Im 20. Jahrhundert wurde die Phraseologie meist unter Ausschuss der Kollokationen und Phraseoterme verstanden. Diese enge Sehweise, in der vor allem die Kollokationen außerhalb jeglicher linguistischen Betrachtung blieben, entsprach nicht der lexikographischen Praxis seit der Renaissance." Heutzutage ist die Gruppe der Phraseologismen viel breiter. Ihren Platz haben in dieser Gruppe neben Kollokationen auch sog. pragmatische Phraseologismen gefunden.
} 
(https://bop.unibe.ch//linguistik-online/issue/view/127), zeugt nicht nur von der immer größeren Rolle der Phraseodidaktik, sondern auch von dem Bedarf an weiteren Forschungsarbeiten zu dieser Problematik. Die Autorin des Beitrags vertritt jedoch die Auffassung, dass Kollokationen bedauerlicherweise immer noch vernachlässigt werden, und zwar nicht nur in der Wortschatzarbeit (vor allem in Bezug auf $\mathrm{DaF}$ ) und Wortschatzdidaktik, sondern auch in anderen Bereichen, die mit dem DaF-Lernen und -Lehren verbunden sind. Auch in der Phraseodidaktik scheinen Kollokationen nicht genügend erkannt worden zu sein bzw. immerhin vernachlässigt zu werden, ${ }^{4}$ obwohl sich diese sprachwissenschaftliche Disziplin in den letzten Jahren schneller entwickelt und aus "dem phraseologischen Dornröschenschlaf" (Kühn 1992: 169) schon aufgewacht ist und nicht mehr "in den Kinderschuhen" steckt (ibd.: 171). Der vorliegende Beitrag setzt sich zum Ziel, auf Faktoren hinzuweisen, die die im Titel des Beitrags formulierte These untermauern und darauf hinweisen, dass Kollokationen als ein vernachlässigtes bzw. nicht genügend erkanntes Gebiet der DaF-/DaZ-Didaktik bezeichnet werden können bzw. müssen. Anschließend werden ein paar Überlegungen skizziert, wie man das Problem der Vernachlässigung der Kollokationen in der DaF-/DaZ-Didaktik beheben könnte.

Ausgegangen wird im Folgenden von dem Begriff Kollokation, der zweifach verstanden und zugleich unterschiedlich aufgefasst wird (Abschnitt 1). Danach wird kurz auf die große Rolle der Kollokationen im Fremdsprachenerwerbsprozess eingegangen (Abschnitt 2). Im Hauptteil des Beitrags werden Indizien genannt, die auf einen hohen Stellenwert eines sprachlichen Phänomens in der Fremdsprachendidaktik hinweisen könnten (Abschnitt 3). Anschließend wird an diesen herausgearbeiteten Indizien der Stellenwert von Kollokationen in der DaF-Didaktik bzw. im DaF-Unterricht überprüft (Abschnitt 4). Dies erfolgt anhand von Analysen und kleinen empirischen Studien, die von der Autorin des vorliegenden Beitrags durchgeführt wurden. Untersucht und analysiert werden die Berücksichtigung der Kollokationen bzw. des kollokationalen Lernens in Handbüchern zur Fremdsprachendidaktik, die Kenntnis dieses Begriffs und dessen Relevanz im Fremdsprachenerwerbsprozess von DaF-Lernenden sowie die Berücksichtigung des kollokationalen Lernens (Wortschatzlernen in Form von Kollokationen) in der selbstständigen Wortschatzarbeit der untersuchten DaF-Lernenden. (frisch gebackene Abiturienten, die das (Auslands)Germanistikstudium aufnehmen).

\section{$1 \quad$ Zum Kollokationsbegriff}

Wie schon oben angedeutet, gibt es keine einheitliche Auffassung des Kollokationsbegriffs, weshalb sich dieser nicht leicht definieren lässt. Das Verständnis dieses Terminus reicht von einer engeren bis zu einer breiteren Deutung. Aus diesem Grund bezeichnet Reder (2006a: 373) Kollokationen als "eine Kategorie mit unscharfen Grenzen". Die ursprüngliche Bezeichnung des uns interessierenden Begriffs ist auf den von Firth eingeführten Terminus collocate zurückzuführen. Dies bedeutet jedoch nicht, dass Firth (1957) diese sprachliche Erscheinung als erster erkannt hat. Schon Porzig (1934) hat auf "wesenhafte Bedeutungsbeziehungen" hingewiesen, wobei darunter außer dem heutigen Kollokationsbegriff im engeren Sinne (die von Hausmann

\footnotetext{
4 Von der bisherigen Vernachlässigung der Kollokationen in der Phraseodidaktik kann die Tatsache zeugen, dass jahrelang in wichtigen Beiträgen zur Phraseodidaktik (vgl. Kühn 1992; Lüger 1997; Ettinger 2002) auf den speziellen Typ der Phraseme - nämlich auf Kollokationen - nicht eingegangen wurde. Man könnte deshalb den Eindruck gewinnen, dass Kollokationen sogar aus dem Blickpunkt der Phraseodidaktik verschwunden sind. Die Analyse der Überlegungen zur Phraseodidaktik in den oben erwähnten Beiträgen zeigt, dass nicht alle didaktischen Vorschläge zu Kollokationen passen. So wird z. B. in Bezug auf idiomatische Phraseme eher für deren passive und viel seltener für deren aktive Beherrschung (diese soll sich nur auf die frequentesten Phraseolexeme beziehen) plädiert. Im Falle der Kollokationen wäre von den DaF-Lernenden meistens ihre aktive Beherrschung zu erwarten. Darüber hinaus treten zahlreiche Kollokationen schon auf dem A1-Niveau (z. B. die Schule besuchen) auf und sollten fast seit dem Beginn des DaF-Lernens als ein sprachliches Phänomen thematisiert werden (was eigentlich auch pragmatische Phraseologismen betrifft). Erfreulich ist jedoch die Tatsache, dass in den neueren phraseodidaktischen Überlegungen Kollokationen endlich schon erwähnt werden (vgl. Ettinger 2013).
} 
1984 propagierte Auffassung des Kollokationsbegriffs) auch andere semantische Beziehungen zwischen den lexikalischen Einheiten subsumiert wurden. Darüber hinaus sind in der Gruppe "lexikalischer Solidaritäten" von Coseriu (1967/1978: 243) auch Kollokationen zu finden.

Bei Kollokationen ${ }^{5}$ handelt es sich um eine Zusammenstellung von Wörtern in einem Syntagma, also um eine syntagmatische Wortverbindung, in der Kollokate, d. h. die einzelnen Elemente einer Kollokation, miteinander kookkurrieren. Kollokationen gehören neben der Valenz zur kombinatorischen Komponente bzw. zum syntagmatisch-kombinatorischen Potenzial einer lexikalischen Einheit (vgl. Wotjak 2007), weswegen diese in verschiedenen Modellen der Komponenten von lexikalischen Einheiten berücksichtigt werden, obwohl sie in jedem Modell anders bezeichnet werden (vgl. Löschmann 1986, 1993; Bohn 1999; Neveling 2004). ${ }^{6}$ Jedoch kann nicht jedes Syntagma automatisch als eine Kollokation gedeutet werden, weil Kollokationen eine spezifische Art des Syntagmas darstellen. Der Begriff Kollokation wird unterschiedlich aufgefasst. Sein Verständnis reicht von einer engen bis zu einer breiten Auffassung, worauf unten näher eingegangen wird.

Die Problematik der Definierung des Kollokationsbegriffs resultiert daraus, dass er einerseits unterschiedlich in der Sprachwissenschaft und in der Fremdsprachendidaktik aufgefasst wird (vgl. dazu Zimmermann 1981). Darüber hinaus unterscheidet sich die Auffassung des Begriffs Kollokation in der Sprachwissenschaft von der in der Phraseologie (Wanzeck 2010: 109). Schneider (1988: 72) weist auf die zweifache Erfassungs- und Auffassungsmöglichkeit der Kollokationen folgendermaßen hin: "Der Kollokationsbegriff vermengt in sich zwei unterschiedliche Aspekte, nämlich den der tatsächlichen Kookkurrenz auf lexikalischer Ebene und den der potenziellen Verträglichkeit auf semantischer Ebene." Zum einen wird von Kollokationen bei jeder Art syntagmatischer Wortverbindungen gesprochen (z. B. hübsches Mädchen). Zum anderen bezieht man diesen Begriff auf "Wortverbindungen mit schwacher semantischer Veränderung" (ibd.). Laut Gładysz (2003: 41) stellen Kollokation nicht nur eine spezifische Wortverbindung, sondern auch eine spezifische Beziehung zwischen den Bestandteilen einer Wortverbindung dar, wodurch sie als eine aus der Verknüpfung von Wörtern resultierende Einheit betrachtet werden können. Kollokationen können darüber hinaus als "inhaltlich geschlossene semantisch-pragmatische Einheiten" (Siepmann 2003: 255), als Wortschatzeinheiten, phraseologische Einheiten (vgl. Reder 2006b, Reder 2011), Halbphraseme (Hausmann 2003) oder als Phraseme bezeichnet werden.

Der Grund für diese Uneinheitlichkeit in der Auffassung dieses Begriffs liegt darin, dass sich das Verständnis der Kollokationen im englischsprachigen und im deutschsprachigen Raum auf unterschiedliche Weise entwickelte. Im englischsprachigen Raum stützt man sich auf die Deutung dieses Terminus im Britischen Kontextualismus, in dem jede Art der frequenten Zusammenstellung von Lexemen bzw. der häufig auftretenden syntagmatischen Verbindungen als Kollokationen bezeichnet wird. (vgl. Sinclair 1991) Dieses Verständnis des Kollokationsbegriffs kann als eine syntaktische Auffassung bezeichnet werden. Für diese breite Auffassung setzen sich z. B. Bahns (1997) und Teubert (2004) ein.

Im deutschsprachigen Raum ist die fremdsprachendidaktisch orientierte Auffassung des Kollokationsbegriffs, die von Hausmann eingeführt und propagiert wurde, am meisten vertreten. ${ }^{7}$

\footnotetext{
${ }^{5}$ Wenn in diesem Artikel der Begriff Kollokation gebraucht wird, dann werden darunter lexikalische Kollokationen (vgl. Scherfer 2008) verstanden, denen Benson (1985) und Benson/Benson/llson (1986 nach Bahns 1996: 17) den Begriff grammatische Kollokationen gegenüber stellen.

${ }^{6}$ Löschmann (1986) positioniert Kollokationen in der grammatisch-kombinatorischen Komponente der lexikalischen Einheit. Laut Löschmann (1993) bilden Kollokationen die Kompatibilitätskomponente, bei Bohn (1999) die kombinatorische Komponente und bei Neveling (2004) die syntagmatische Komponente der lexikalischen Einheit.

${ }^{7}$ Diese kann nach Müller (2011: 14) eine bedeutungsorientierte Auffassung des Kollokationsbegriffs genannt werden.
} 
Hausmann (1984: 399-402) versteht unter Kollokationen nur solche syntagmatischen Wortverbindungen, bei denen die semantisch autonome Basis, die mit anderen Wörtern kolloziert, den Einsatz von bestimmten weiteren sprachlichen Elementen verlangt bzw. bestimmt, was als nächstes Element der Wortkette stehen soll. Das zweite Gliedelement bildet der Kollokator, der semantisch an die Basis gebunden ist. Der Kollokator ergänzt im Kontext die Basis und sie verschmelzen zu einer festen Wortverbindung, wodurch jede Kollokation eine spezielle Bedeutung bekommt. In manchen Kollokationen kann der Kollokator weder beliebig gewählt noch durch ein Synonym ersetzt werden (z. B. Zähne putzen vs. *Zähne waschen ${ }^{8}$; starker Raucher vs. *kräftiger Raucher; neue Kartoffeln vs. *junge Kartoffel; ein Amt bekleiden vs. *ein Amt anziehen), wobei dieser seine primäre Bedeutung nicht verliert. In anderen Kollokationen kann der Kollokator seine primäre Bedeutung verlieren. In diesem Fall spezifiziert dieser nur die Bedeutung der Kollokationsbasis (z. B. ein Risiko eingehen, eine Entscheidung treffen). ${ }^{9} \mathrm{Da}$ gerade diese Art der spezifischen syntagmatischen Wortverbindungen den Fremdsprachenlernenden große Probleme bereiten kann, weil in diesen Kollokationen die Wörter nicht beliebig kreativ zusammenstellbar sind, vertritt Hausmann die Meinung, dass nur diese Art der Kollokationen (Kollokationen im engeren Sinne) als solche bezeichnet werden sollte bzw. darf. Seiner Ansicht nach ist "der basisbezogene Kollokationsbegriff [...] der engere, der merkmalreichere, der elaboriertere, der genauere, der funktionalisiertere, der anwendungsbezogenere, folglich der unverzichtbarere" (id. 2004: 321). Diesen engen Kollokationsbegriff hält Hausmann für die Fremdsprachendidaktik für den besten und geeignetsten. Deshalb fordert er die Linguisten dazu auf, sich für den breit aufgefassten Kollokationsbegriffs eine neue Bezeichnung auszudenken.

\section{Rolle der Kollokationen und kollokationalen Lernens im DaF-Lernprozess}

Die Relevanz des kollokationalen Lernens resultiert daraus, dass Kollokationen eine sehr frequente sprachliche Erscheinung darstellen, die sowohl im mündlichen als auch im schriftlichen Gebrauch auftritt. Ihre Form ist leider unauffällig bei der Rezeption, ${ }^{10}$ weil sie nicht gegen syntaktische und selten gegen semantische Kompatibilitätsregeln verstoßen. Die Ursache für diese Unauffälligkeit könnte auch in der stiefmütterlichen Behandlung der Phraseologie - darunter auch der Kollokationen - im Muttersprachenunterricht liegen. Kollokationen sind leicht erschließbar und werden als ein normaler Sprachgebrauch empfunden. Der Fremdsprachenlernende versteht (größtenteils) sofort die Bedeutung einer Kollokation, wenn er die Bedeutung der Kollokationsbasis kennt und erkennt. Deshalb richtet er beim Rezeptionsprozess seine Aufmerksamkeit nicht auf den Kollokator, sondern auf die die Bedeutung tragende Kollokations-

\footnotetext{
${ }^{8}$ Bei den mit * gekennzeichneten Beispielen handelt es sich um inkorrekte Formen, die keine Kollokationen darstellen, die jedoch als solche von den DaF-Lernenden oft gebildet und gebraucht werden.

${ }^{9}$ In der Diskussion um den Kollokationsbegriff wird darüber diskutiert, ob Funktionsverbgefüge (FVG) als Kollokationen fungieren können bzw. sollen. Einerseits werden FVG den Kollokationen zugerechnet (vgl. Laretta Zulategui 2006), andererseits aus dieser Gruppe ausgeschlossen (vgl. Wojtak/Heine 2005). Gładysz (2003) zählt FVG zu den Kollokationen, wobei er die unscharfe Grenze zwischen den beiden Gruppen von Phrasemen zum Ausdruck bringt: "Die Grenzen zwischen beiden Erscheinungen [= FVG und Kollokationen] bleibt also unscharf. Nach einer weiten Auffassung des Kollokationsbegriffs können Funktionsverbgefüge in den Kollokationsbereich integriert werden." (ebenda: 85) Im Folgenden werden FVG der Gruppe der Kollokationen zugerechnet, da in dieser spezifischen Wortverbindung das Substantiv (als Kollokationsbasis) seine primäre Bedeutung beibehält und das Verb, das gerade seine primäre Bedeutung verliert, nur die Rolle des Funktionsverbs annimmt. In dieser festen Wortverbindung kann das Verb nicht frei zu einem Substantiv gewählt werden. Dies ist gerade das charakteristische Merkmal der Kollokationen. In dem bis dahin einzigen für DaF-Lerner in Buchform erschienenen Wörterbuch der deutschen Kollokationen (Quasthoff 2011) werden FVG auch aufgenommen, was als zusätzliches Argument für die Aufnahme der FGV in die Gruppe der Kollokationen gelten kann.

${ }^{10}$ Handwerker (2010: 249) verweist darauf, dass Kollokationen für den Muttersprachler auch nicht auffällig sind. Er ist sich der Kollokationen nicht bewusst. Erst durch die kontrastive Betrachtung der Muttersprache mit der zu lernenden Fremdsprache kann sich der Muttersprachler der Existenz der Kollokationen bewusst werden.
} 
basis. Dabei konzentriert er sich auf den Inhalt und nicht auf die sprachliche Form, denn Kollokationen erscheinen dem Fremdsprachenlernenden als eine freie Wortverbindung (vgl. Handwerker 2010: 249). Da Kollokationen von Sprache zu Sprache variieren, d. h. in der jeweiligen Sprache anders realisiert werden (können), stellen sie insbesondere in der Sprachproduktion oft eine Fehlerquelle dar (obwohl sie in der Rezeption meistens nicht problematisch sind). Der Fremdsprachenlernende kann nämlich nicht selbstständig eine Kollokation bauen bzw. diese aus einer (Fremd)Sprache in die andere übertragen, denn diese sind nicht selbstständig produzierbar, sondern als Mehrworteinheiten reproduzierbar. Diese lassen sich auch nicht voraussagen, denn eine Kollokation kann in zwei Sprachen einen anderen Kollokator haben. Die Unvorhersagbarkeit der Kollokationen ist darauf zurückzuführen, dass diese ein Element der Sprachnorm und nicht eins der Regeln darstellen (Tomas 2008: 79). Aus diesem Grunde muss der Fremdsprachenlernende zur korrekten Anwendung von Kollokationen diese als eine Einheit (Kollokator mit seiner Basis) abspeichern und bei der Sprachproduktion diese als solche aktivieren und gebrauchen.

Wenn man mit Stein (1995) annimmt, dass das Deutsche eine formelhafte Sprache ist, in der viele Sprechabsichten bzw. viele Kommunikate nicht aus losen Wörtern, sondern aus fertigen Schablonen bzw. Formeln zusammengebaut werden, dann bilden Kollokationen auch solch ein formelhaftes Element der Sprache. Deshalb stellen sie "Halbfertigprodukte" (vgl. Hausmann 1984: 398) dar, die man in eine Äußerung einflechten kann bzw. muss. Als vorgefertigte Strukturen sind sie zweifelsohne hilfreich in der mündlichen Sprachproduktion, denn ihr Einsatz beeinflusst positiv den Redefluss und führt zur Natürlichkeit des fremdsprachlichen Ausdrucks (vgl. Handwerker 2010). Beim Internalisieren der Kollokationen erwerben Fremdsprachenlernende laut Hill (1999: 5) gleichzeitig ein Betonungsschema dieser Art von Chunks, so dass sie nicht die einzelnen Wörter als separate Einheiten betonen, sondern ein Betonungsschema einer Kollokation übernehmen. Dabei können sie sich mehr auf den Inhalt als auf die Suche nach einem Kollokator konzentrieren.

Zöfgen (2001) führt drei wichtige Argumente dafür an, warum Kollokationen im FU ein wichtiger Platz eingeräumt werden bzw. ihnen viel mehr Aufmerksamkeit geschenkt werden sollte. Dabei handelt es sich erstens um den sprachstrukturellen Aspekt (ein hoher Anteil des Sprachgebrauchs ist "durch vorgefertigte Äußerungen stark routiniert" (Stubbs 1997: 153 nach Zöfgen 2001: 272), zweitens um die lern- und gedächtnispsychologische Dimension (Struktur des mentalen Lexikons, unter dessen Netzen ${ }^{11}$ gerade syntagmatische Verknüpfungen zu finden sind; Lernen von Kollokationen entspricht den Erfordernissen des mentalen Lexikons) und die empirische Evidenz (eine der Hauptursachen von Kollokationsfehlern ist die Interferenz aus der L1).

Kollokationen stellen nicht nur Halbfertigprodukte dar, sondern werden auch als Sinneinheit und als Übersetzungseinheit bezeichnet. Im ersten Fall handelt es sich darum, dass die Bedeutung einer kollokablen Wortverbindung nicht aus der Bedeutung der Einzelwörter, sondern gerade aus der Verbindung dieser Elemente (Kollokator spezifiziert die Bedeutung der Kollokationsbasis) resultiert, was Teubert (2004: 522) folgendermaßen formuliert: "In einer Sinneinheit spielen die Einzelwörter keine Rolle mehr. Nur die Einheit als solche hat eine Bedeutung". ${ }^{12}$

\footnotetext{
11 Mehr zu den Wörternetzen im mentalen Lexikon findet sich bei Kielhöfer (1994) und Neveling (2004).

12 Diese Erklärung bezieht sich auf diese spezielle Art der Kollokationen, in denen (insbesondere bei den Substantiv-Verb-Kollokationen) die Kollokationsbasis ihre Bedeutung beibehält und der Kollokator seine primäre Bedeutung in der Kollokation verliert und nur die Bedeutung der Kollokationsbasis spezifiziert, wie z. B. in folgenden Kollokationen: Geld abheben, die Prüfung ablegen, einen Eid ablegen, Verantwortung tragen, Kosten tragen. Wenn der Fremdsprachenlernende eine Kollokation nicht als solche wahrnimmt, beginnt er, sich auf die Einzelwörter dieser konventionellen Wortverbindung zu konzentrieren. In dieser Art der Kollokationen können die Er-
} 
Des Weiteren bilden Kollokationen eine Übersetzungseinheit, denn im Übersetzungsprozess der Kollokationen sollen nicht deren einzelne Bestandteile separat in die Fremdsprache übertragen werden, sondern für eine Kollokation in der Ausgangssprache soll in einer Fremd- bzw. Zielsprache eine Äquivalenz gefunden werden.

Es ist nicht ausgeschlossen (der Autorin sind keine empirischen Studien bekannt, die dies untersuchen würden und bestätigen könnten), dass die Bewusstheit dieses sprachlichen Phänomens den Rezeptionsprozess verschiedener Wortverbindungen und Zusammenstellungen begünstigen könnte. Dabei muss betont werden, dass es sich nicht um die Kenntnis der jeweiligen Kollokation bzw. um den Abruf der schon gespeicherten Kollokationen handelt, sondern um das Wissen darüber, dass es diese spezifische Art der Syntagmen, d. h. Kollokationen im Deutschen (aber auch in den meisten Sprachen) gibt. Dank dem Kollokationsbewusstsein könnten DaF-Lernende ihnen unbekannte Kollokationen als solche erkennen, so dass sie nicht nach der Bedeutung der Bestandteile einer zum ersten Mal begegneten Kollokation suchen, sondern die Gesamtbedeutung der ganzen konventionellen Wortverbindung zu erschließen versuchen.

\section{Indizien für einen hohen Stellenwert eines sprachlichen Phänomens im DaF-Un- terricht}

Im Titel dieses Beitrags wurde die These aufgestellt, dass Kollokationen in der DaF-/DaZ-Didaktik vernachlässigt werden. Welche Indizien verweisen darauf, dass ein Begriff in der Fremdsprachendidaktik wichtig ist? Im Folgenden wurde davon ausgegangen, dass auf einen hohen Stellenwert eines Phänomens bzw. Begriffs in der Fremdsprachendidaktik folgende Faktoren verweisen können, die unten stichwortartig präsentiert werden:

1. Explizite Definition des Begriffs Kollokation und Kollokationskompetenz (theoretische Grundlagenforschung),

2. Erwähnung der Begriffe Kollokation und Kollokationskompetenz in Handbüchern zur DaF-/DaZ-Didaktik (DaF-Lehrerausbildung), ${ }^{13}$

3. Empirische Studien zu Kollokationen bzw. zur Entwicklung der Kollokationskompetenz aus der Sicht der Fremdsprachenlehr- und -lernforschung (empirische Forschung),

4. Bewusstheit (der Rolle) der Kollokationen und des kollokationalen Lernens im DaFLehrprozess seitens der Lehrkräfte und im DaF-Lernprozess seitens der DaF-Lernenden (Unterrichtsprozess),

5. Erwähnung des Begriffs Kollokationen bzw. Bewusstmachung der Existenz von diesem sprachlichen Phänomen in Lehrwerken (Konzeption der Lehrwerke),

6. Übungsreihen zu Kollokationen und zur Entwicklung der Kollokationskompetenz in Lehrwerken für verschiedene Alters- und Niveaustufen sowie in zusätzlichen Lehr- und Selbstlernmaterialien (Übungen in Lehrwerken und Lehrmaterialien),

7. Einbeziehung von Kollokationen bzw. Überprüfung der rezeptiven und/oder produktiven Kenntnis in Prüfungen für DaF (Abiturprüfungen, DaF-Zertifikate).

\footnotetext{
schließung der Bedeutung von einzelnen Elementen der kollokativen Verbindung und der Versuch des Zusammenfügens von den Bedeutungen der Einzelglieder der Kollokationen die DaF-Lernenden irreführen. Dies betrifft auch die Bedeutung der FVG.

13 Man könnte hier zwar einwenden, dass diese zwei Indizien nicht direkt auf die Desiderata der Kollokationsproblematik hinweisen. Im Folgenden wird die Ansicht vertreten, dass ein aus der Sicht der Fremdsprachendidaktik und der Fremdsprachenlehr- und -lernforschung wichtiges Sprachphänomen in den Handbüchern zur Didaktik der jeweiligen Fremdsprache berücksichtigt werden muss.
} 


\section{4 Überprüfung des Stellenwerts von Kollokationen in der DaF-/DaZ-Didaktik}

Im folgenden Abschnitt des Artikels wird ausführlich auf die ersten vier Punkte der oben genannten Indizien eingegangen, wobei die Faktoren drei und vier an einigen Beispielen exemplarisch analysiert werden. Aus Platzgründen werden die letzten drei Punkte in diesem Beitrag nicht thematisiert, weil deren Behandlung separate Beiträge gewidmet werden könnten/sollten. Ausgegangen wird von der Suche nach der Definition des Begriffs Kollokationskompetenz (siehe 4.1). Danach folgt die Analyse der Thematisierung der Kollokationen und der Berücksichtigung des Kollokationsbegriffs bzw. Kollokationsphänomens in Nachschlagewerken zur DaF-Didaktik (Kap. 4.2). Anschließend wird auf die Präsentation der empirischen Forschungen bezüglich der Kollokationen eingegangen, wobei besonderer Nachdruck auf die glottodidaktisch orientierte DaF-Kollokationsforschung gelegt wird (Kap. 4.3). Abgeschlossen wird dieses Kapitel mit der Erfassung der Bewusstheit der Existenz von Kollokationen seitens der DaFLernenden sowie mit der Darstellung von Ergebnissen der empirischen Forschung bezüglich der Berücksichtigung des kollokationalen Lernens in der selbstständigen Wortschatzarbeit der DaF-Lernenden (siehe 4.4).

\subsection{Zum Begriff "Kollokation" und "Kollokationskompetenz"}

Problematisch ist in der deutschsprachigen Fremdsprachendidaktik nicht nur die Auffassung des Kollokationsbegriffs, der in der überwiegenden Mehrheit der Nachschlagewerke zur DaF/DaZ-Didaktik nicht näher erläutert wird, worauf schon oben hingewiesen wurde. Auch die Deutung des Begriffs Kollokationskompetenz kann gewisse Probleme bereiten. Zum einen wird dieser in keinem der analysierten Handbücher und Lexika erklärt. Zum anderen wird dieser Terminus in vielen englisch- (vgl. Hill 1999) und deutschsprachigen Forschungsarbeiten (vgl. Reder 2002, 2006; Holderbaum 2003; Forkl 2010; Rössler 2010; Müller 2011) gebraucht, aber in keiner dieser Publikationen wird der Versuch gewagt, den Begriff Kollokationskompetenz explizit zu erklären. Dies kann verwundern, weil auch in wissenschaftlichen Arbeiten, in deren Titeln der Begriff "Kollokationskompetenz" auftritt, dieser auch nicht explizit definiert wird. Davon, dass die Kollokationskompetenz keine einheitliche Größe darstellt, sondern auch aus Teilkompetenzen besteht, zeugen die in den oben erwähnten Forschungsarbeiten gebrauchten Termini produktive Kollokationskompetenz und rezeptive Kollokationskompetenz.

Es besteht jedoch keine Sicherheit, ob nur diese zwei Subkompetenzen unter den Terminus Kollokationskompetenz zu subsumieren sind. Diese stützt sich einerseits auf Überlegungen von Lütge (2000) und Müller (2011), die eine große Rolle des Kollokationsbewusstseins bei der Entwicklung der Kollokationskompetenz betonen. Zu überlegen wäre also die Aufnahme einer weiteren Subkompetenz der Kollokationskompetenz (vgl. Fandrych $2008^{14}$ und Targońska/Stork 2013). Festzuhalten ist allerdings die Notwendigkeit der Spezifizierung des fremdsprachendidaktisch orientierten Kollokationsbegriffs und des Terminus Kollokationskompetenz.

\subsection{Kollokation und Kollokationskompetenz in Handbüchern zur DaF/DaZ-Didaktik und Fremdsprachendidaktik}

Wie oben erläutert, wird im Folgenden davon ausgegangen, dass ein Indiz dafür, ob ein Begriff bzw. eine sprachliche Erscheinung als relevant in der Fremdsprachendidaktik betrachtet werden kann, die Thematisierung des jeweiligen Begriffs in gängigen Handbüchern und Lexika zur Fremdsprachendidaktik bzw. zur Didaktik der jeweiligen Fremdsprache ist. Aus diesem Grunde wurden einige solche Nachschlagewerke untersucht, wobei sowohl ältere als auch neuere DaF-

\footnotetext{
${ }^{14}$ Dabei handelt es sich um Fandrychs Appell, alle Kompetenzen als dreigliedrig aufzufassen (rezeptive, produktive und reflexive Kompetenzen).
} 
Didaktik-Handbücher einer Analyse unterzogen wurden. Dabei wurden drei Elemente untersucht:

- Ist der Kollokationsbegriff im Register des Handbuchs zu finden?

- Wird den Kollokationen ein separates Kapitel bzw. Unterkapitel gewidmet?

- Wird auf diesen Begriff und seine Relevanz in Kapiteln zur Wortschatzarbeit bzw. zum Wortschatzlernen eingegangen?

Die Ergebnisse der Analyse wurden in der Tabelle 1 festgehalten, wobei die Handbücher und Nachschlagewerke chronologisch nach dem Erscheinungsjahr geordnet wurden.

\begin{tabular}{|l|c|c|c|}
\hline & $\begin{array}{l}\text { Begriff Kol- } \\
\text { lokation } \\
\text { im Register }\end{array}$ & $\begin{array}{l}\text { Thema des } \\
\text { Kapitels bzw. } \\
\text { Unterkapitels }\end{array}$ & $\begin{array}{l}\text { Erwähnung } \\
\text { des Begriffs } \\
\text { in Kapiteln }\end{array}$ \\
\hline $\begin{array}{l}\text { Didaktik des Fremdsprachenunterrichts } \\
\text { (Deutsch als Fremdsprache). (Dessel- } \\
\text { mann/Hellmich 1986) }\end{array}$ & - & - & - \\
\hline $\begin{array}{l}\text { Deutsch lehren. Grundwissen für den Un- } \\
\text { terricht in Deutsch als Fremdsprache. } \\
\text { (Heyd 1991) }\end{array}$ & - & - & - $/-$ \\
\hline $\begin{array}{l}\text { Handbuch Fremdsprachenunterricht. } \\
\text { (Bausch/Christ/Krumm 1995) }\end{array}$ & - & - & - \\
\hline $\begin{array}{l}\text { Deutsch als Fremdsprache - Eine Didaktik. } \\
\text { (Storch 1999) }\end{array}$ & - & - & - \\
\hline $\begin{array}{l}\text { Deutsch als Fremdsprache. Ein internatio- } \\
\text { nales Handbuch. (Helbig/Henrici/Krumm } \\
\text { 2001) }\end{array}$ & - & - & - \\
\hline $\begin{array}{l}\text { Einführung in die Didaktik des Unterrichts } \\
\text { Deutsch als Fremdsprache mit Videobei- } \\
\text { spielen. (Henrici/Riemer 2001) }\end{array}$ & - & - & - \\
\hline $\begin{array}{l}\text { Deutsch als Fremdsprache. Eine Einfüh- } \\
\text { rung. (Huneke/Steinig 2005) }\end{array}$ & - & - & - \\
\hline $\begin{array}{l}\text { Fremdsprachenerwerb-Fremdsprachendi- } \\
\text { daktik. (Roche 2008) }\end{array}$ & - & - & - \\
\hline $\begin{array}{l}\text { Fortbildung für Kursleitende Deutsch als } \\
\text { Zweitsprache. (Kaufmann et al. 2008) }\end{array}$ & - & - & - \\
\hline $\begin{array}{l}\text { Fachlexikon Deutsch als Fremd- und } \\
\text { Zweitsprache. (Barkowski/Krumm 2010) }\end{array}$ & - & - & - \\
\hline $\begin{array}{l}\text { Fremdsprachendidaktik. (Decke-Cor- } \\
\text { nill/Köster 2010) }\end{array}$ & - & - & - \\
\hline $\begin{array}{l}\text { Deutsch als Fremdsprache - Spracher- } \\
\text { werblich reflektierte Unterrichtspraxis. } \\
\text { (Koeppel 2010) }\end{array}$ & - & - \\
\hline $\begin{array}{l}\text { Deutsch als Fremdsprache. Ein internatio- } \\
\text { nales Handbuch. (Krumm et al. 2010) }\end{array}$ & - & - \\
\hline $\begin{array}{l}\text { Metzler Lexikon Fremdsprachendidaktik. } \\
\text { (Surkamp 2010) }\end{array}$ & - & - \\
\hline
\end{tabular}

Tab. 1: Berücksichtigung des Kollokationsbegriffs in ausgewählten Handbuchern zur DaF-Didaktik. 
Schon beim ersten Blick auf diese Tabelle fällt auf, dass der Begriff Kollokation in Handbüchern zur DaF-/DaZ-Didaktik nicht berücksichtigt wird. Zwar ist in fünf der analysierten Handbüchern (vgl. Heyd 1991; Kaufmann et al. 2008; Barkowski/Krumm 2010; Koeppel 2010; Krumm et al. 2010) der untersuchte Begriff im Register zu finden, aber dies allein kann noch nicht als ein Hinweis auf die Relevanz dieses Begriffs gedeutet werden. In Kapiteln zum Wortschatzlernen bzw. zur Wortschatzarbeit im DaF-/DaZ-Unterricht werden Kollokationen in den meisten analysierten Nachschlagewerken gar nicht erwähnt. Nur in vereinzelten Büchern werden sie sehr kurz angesprochen und zwar bei Heyd (1991); Helbig/Henrici/Krumm (2001); Huneke/Steinig (2005); bei Decke-Cornill/Köster (2010) sowie bei Koeppel (2010). Heyd (1991: 75) gebraucht den Kollokationsbegriff im Kapitel zur Arbeit an Texten im FU, wo sie auf die Rolle der syntagmatischen Strukturen (darunter gerade der Kollokationen) und Idiome bei der Spracherlernung hinweist. Bei Kaufmann et al. (2008: 33) wird der Kollokationsbegriff in dem an das Kapitel "Wortschatzvermittlung im DaZ-Unterricht" angehängten Glossar erklärt. Den beiden Erklärungen des Kollokationsbegriffs von Heyd (1991: 276) und MeerholzHärle (2008: 33) liegt das weite Verständnis des Kollokationsbegriffs zugrunde. Die erste deutet diese als "eine feste Wortkombination (ein Sonderfall des Syntagmas)" (Heyd 1991: 276) und die zweite versteht sie als eine "Bezeichnung für häufig zusammen auftretende Wörter, die semantisch - also von ihrer Bedeutung her - verbunden sind, z. B. Katze - miauen, Tag - hell" (Meerholz-Härle 2008: 33). Bei Decke-Cornill/Küster (2010: 164) ist ein Hinweis auf Kollokationen bezüglich des mentalen Lexikons zu finden, wo Kollokationen als eine Art der mentalen Wortverbindung und als eine behaltensförderliche Art des Wortschatzlernens dargestellt werden. Dort wird auch darauf hingewiesen, dass neue Wörter nicht als Einzelwörter eingeführt und geübt werden, sondern diese in Kollokationen erscheinen sollten. Darüber hinaus wird die Rolle der Kollokationen für die aktive Sprachverwendung angesprochen. (ibd.: 169). Huneke/Steinig (2005: 149) verweisen auf die Rolle der Lehrkraft, die bei der Semantisierung die neuen Wörter nicht nur paradigmatisch, sondern auch syntagmatisch einordnen soll.

Nur im Falle des Internationalen Handbuchs Deutsch als Fremdsprache von Krumm et al. (2010) kann keine Rede von der Vernachlässigung der Kollokationen als einer sprachlichen Erscheinung sein. In dieser Publikation wird zum ersten Mal ein separates Kapitel den Kollokationen gewidmet. Zwar heißt dieses von Handwerker (2010: 246-255) verfasste Unterkapitel Phraseologismen und Kollokationen, aber leider wird dort nur ein Absatz Kollokationen gewidmet. Dies ist jedoch schon als eine positive Erscheinung aufzufassen. Darin beschäftigt sich Handwerker mit dem Begriff Kollokation und seinen Anfängen, mit dem Bau der Kollokationen und mit Methoden der Identifizierung von Kollokationen. Sie verweist auch auf die Festigkeit der Phraseologismen und Kollokationen, weswegen sie diese als "vorgefertigte Sequenzen" (ibd.: 249) bezeichnet. Die Festigkeit der Kollokationen resultiert aus der gegenseitigen Vorhersagbarkeit der Elemente der jeweiligen Kollokation. Darüber hinaus betont Handwerker die Relevanz der Abspeicherung von Kollokationen für die lernersprachliche Produktion. ${ }^{15}$ Aber auch bei Koeppel (2010), der zwar den Kollokationen kein separates Unterkapitel gewidmet hat, wird dieser uns interessierende Begriff weitgehender thematisiert. Im Kapitel zum Wortschatz verweist Koeppel darauf, dass die Rolle der Mehrwortbenennungen und darunter Kollokationen im Wortschatzerwerb schon erkannt worden ist. Darüber hinaus betont er, dass zum lexikalischen Wissen zur lexikalischen Einheit die Kenntnis der syntagmatischen Beziehungen gehört, weswegen Lehrkräfte bei der expliziten Bedeutungsvermittlung die kollokationalen Verknüpfungen bei der Tafelanschrift berücksichtigen sollten.

\footnotetext{
15 Im DaF-Handbuch von Krumm et al. (2010) werden Kollokationen auch im Kapitel von Tschirner (2010) zum Wortschatz (Kollokationen als syntagmatische Wortverbindungen) und in dem von Kühn (2010) verfassten Kapitel zu den Wörterbüchern fokussiert (Notwendigkeit der Konsultation von semantisch-syntaktischen Problemen in Wörterbüchern, die Notwendigkeit besserer Kollokationsangaben in diesen Nachschlagewerken).
} 
Aus der Analyse der DaF-/DaZ-Didaktik-Handbücher geht hervor, dass dieses wichtige Sprachphänomen, nämlich Kollokation, im ungenügenden Maße in diesen Werken behandelt wird. Zwar wird in einigen Handbüchern dieser Begriff gebraucht und - allerdings kurz - erläutert, aber auf seine Relevanz im Lehr- und Lernprozess des Deutschen als Fremd- bzw. -zweitsprache wird nicht eingegangen. Als ein Desiderat kann also der Hinweis auf die Relevanz der Behandlung der Kollokationen und die Entwicklung der Kollokationskompetenz bezeichnet werden.

\subsection{Fremdsprachendidaktisch bzw. fremdsprachenerwerbsorientierte Kollokations- forschung}

Zwar erfreuen sich seit einigen Jahrzehnten Kollokationen als Gegenstand der wissenschaftlichen Forschung immer größerer Beliebtheit, aber es handelt sich insbesondere um sprachwissenschaftliche, lexikographische und dolmetscherische/übersetzerische Kollokationsforschung und viel weniger um die fremdsprachendidaktisch- bzw. fremdsprachenerwerbsorientierte Kollokationsforschung. Ein großes Desiderat ist in Bezug auf die Erforschung der Rolle der Kollokationen in der Wortschatzarbeit, beim Wortschatzerwerb im DaF-/DaZ-Unterricht, bzw. bezüglich der Untersuchung der Entwicklung von der Kollokationskompetenz festzuhalten. Um eine Diskrepanz zwischen der linguistischen, lexikographischen und dolmetscherischen einerseits und der fremdsprachendidaktisch orientierten Kollokationsforschung andererseits aufzuzeigen, wird im Folgenden kurz auf die erste Gruppe der Forschungsarbeiten eingegangen.

Den Gegenstand der sprachwissenschaftlichorientierten Kollokationsforschung bildet die Beschreibung des Kollokationsbegriffs, insbesondere dessen syntaktischer und semantischer Eigenschaften sowie die Klassifikation der Kollokationen. Hausmann (1984) differenzierte drei Arten der nicht-fixierten Wortverbindungen ${ }^{16}$ aus der Sicht der Fremdsprachendidaktik, zu denen er neben den Kollokationen auch Ko-Kreationen und Konter-Kreationen zählte. In der Forschungsliteratur sind unterschiedliche Klassifikationen der Kollokationen zu finden. (vgl. Kromann 1989; Tomas 2008; Konecny 2010). Darüber hinaus geht die sprachwissenschaftliche Forschung in Richtung bilingualer vergleichender Untersuchungen von Kollokationen in verschiedenen Sprachkonstellationen. Untersucht wurden in Bezug auf Kollokationen u. a. solche Sprachenpaare wie Deutsch-Polnisch (Gładysz 2003) und Deutsch-Tschechisch (Kratochvilova 2004). Mit der Klassifizierung italienischer Kollokationen befasste sich Konecny (2010). Da Kollokationen eine wichtige Fehlerursache bei der Sprachmittlung darstellen (vgl. Holderbaum/Kornelius 2001), wurden Kollokationen als Übersetzungsproblem auch wissenschaftliche Arbeiten bzw. Beiträge gewidmet (vgl. Białek 2009; Grauer 2009; Żmudzki 2000; Gładysz 2002).

Einen wichtigen Bereich stellt die lexikographische Kollokationsforschung dar. Dabei wurden einerseits Überlegungen zur Art und Weise der Darstellung von Kollokationen in Wörterbüchern angestellt. Diskutiert wurde, wie Kollokationen zu erfassen und wo sie zu vermerken sind - beim Kollokator bzw. bei der Basis (vgl. Hausmann 1984; Schneider 1988; Pöll 1997; Steyer 2008; Teubert 2004; Tomas 2008). Inzwischen wurden Konzeptionen für lexikographische elektronische Wörterbücher dargestellt (vgl. Hollós 2004, 2008; Duurčo 2010; Geyken 2011; Klosa/Storjohann 2011). Eine andere Gruppe der lexikographischorientierten Kollokationsforschung bezieht sich auf die Analyse der allgemeinen Wörterbücher bzw. verschiedener Arten der Kollokationswörterbücher des Englischen hinsichtlich der Erfassung von Kollokationen (z. B. Bahns 1987, 1994, 1996. Vgl. Holderbaum 2003; Leśniewska 2008). Analysiert wurden auch Fachwörterbücher (vgl. Bergenholtz/Tarp 1994) sowie einsprachige Wörterbü-

\footnotetext{
16 Diese stellte er den fixierten Wortverbindungen gegenüber, zu denen er Idiome, Routineformeln, Slogans, Sprichwörter zählt. Diese weisen eine erstarrte lexikalische Form auf (vgl. Hausmann 1984: 399).
} 
cher des Französischen (vgl. Zöfgen 2008). In Bezug auf DaF sind zwei Analysen von Lernerwörterbüchern von Köster und Neubauer (2002) und Möhring (2011) zu nennen. Darüber hinaus ist in Bezug auf DaF das Erscheinen des Kollokationswörterbuchs von Quasthoff (2011) festzuhalten, das auf der Grundlage des im Projekt Deutscher Wortschatz (www.wortschatz.uni-leipzig.de) entstandenen Korpus erarbeitet wurde, in dem jedoch neben den Kollokationen im engeren Sinne auch viele syntagmatische Wortverbindungen verzeichnet werden. ${ }^{17}$ Momentan entsteht auch in der Schweiz ein elektronisches Kollokationenwörterbuch, das in der noch nicht fertigen Fassung im Internet abzurufen ist (http://colloc.germa.unibas.ch/web/suche/). Dort sind jedoch im Moment viele syntagmatische Wortverknüpfungen zu finden (Kollokationen im weiteren Sinne), wobei bedauerlicherweise die typischsten Kollokationen im engeren Sinne noch nicht erfasst wurden. Erwähnenswert ist auch eine Webseite (http://www.kollokation.at), die im Zusammenhang mit einem Projekt zum Vergleich der deutschen und italienischen Kollokationen entstanden ist, auf der verschiedene Arten der Kollokationstypen sowohl unter dem morpho-syntaktischen als auch unter dem semantisch-begrifflichen Aspekt unterschieden werden.

Wie oben gezeigt wurde, bezieht sich Kollokationsforschung vor allem auf die drei Bereiche Linguistik, Lexikographie und Dolmetschen. Ein großes Desiderat muss jedoch in der fremdsprachendidaktisch- bzw. fremdsprachenerwerbsorientierte DaF-Kollokationsforschung festgehalten werden. Die ersten empirischen den Kollokationen gewidmeten Studien bezogen sich auf Englisch als Fremdsprache (vgl. Marton 1977; Bahns/Sibilis 1992 und Gabryś-Biskup 1990). Den Mangel an solchen fremdsprachendidaktisch orientierten DaF-Kollokationsforschungen bekräftigt auch die von Storjohann (2011) zusammengestellte Bibliographie zu Kollokationen, in der sehr viele Literaturangaben zum Begriff Kollokation, zu Kollokationen und Semantik, zu Kollokationen in der Phraseologie, zur empirischen, korpusgestützten Kollokationsforschung, zu Kollokationen in Fach- und Wissenschaftssprachen, in der Lexikographie und Übersetzungswissenschaft, jedoch nur wenige wissenschaftliche Arbeiten zu Kollokationen beim Spracherwerb ausfindig gemacht werden konnten. Davon bezieht sich die überwiegende Mehrheit der Publikationen zu Kollokationen im (Fremd)Spracherwerbsprozess auf Englisch als Fremdsprache und lediglich eine Hand voll auf Deutsch als Fremdsprache bzw. Französisch als Fremdsprache.

Glottodidaktisch ${ }^{18}$ orientierte Studien zu Kollokationen im DaF-Lehr- und -Lernprozess stellen immer noch eine Seltenheit dar. Aus Platzgründen können diese im Folgenden nur in Umrissen beschrieben werden. Die erste der Autorin des vorliegenden Beitrags bekannte kleine Studie zur Kollokationskompetenz der DaF-Lernenden stammt von Reder (2001), die ähnlich wie Bahns/Sibilis (1992), den Erwerb von neuen Kollokationen mittels Lesen von einer Lektüre

\footnotetext{
${ }^{17}$ Eigentlich handelt es sich bei diesem Nachschlagewerk um das Wörterbuch der Syntagmen, aus dem schwer zu entnehmen ist, ob man die Wörter frei zusammenstellen kann, ob es sich bei manchen Wortverbindungen um Kollokationen handelt. Diesem Nachschlagewerk kann z. B. entnommen werden, dass sowohl die Verbindungen "eine Antwort geben" und "eine Antwort erteilen" (Quasthoff 2011: 33) als auch "den Unterricht erteilen" und "Unterricht geben" (ibd.: 473) möglich sind. (Dabei geht aus den theoretischen Überlegungen zum Kollokationsbegriff hervor, dass in Kollokationen Kollokatoren durch synonyme Verben nicht ersetzt werden können und in diesen Beispielen ist das möglich). Dies kann auch daran liegen, dass dieser Zusammenstellung von Kollokationen eine sehr breite Auffassung des Kollokationsbegriffs zugrunde liegt, denn der Autor versteht unter Kollokationen "Paare von Wörtern, die typischerweise zusammen auftreten" (ibd.: Vorwort). Andererseits ist das Wörterbuch auf der Korpusgrundlage, d. h. auf der Grundlage authentischer Texte entstanden, in denen nach signifikanten Nachbarschaftskookkurrenzen gesucht wurde, wobei die Kollokations-Kandidaten für dieses Wörter manuell bearbeitet wurden.

18 Der Begriff Glottodidaktik existiert in einigen Sprachen, wobei in Polen Glottodidaktik sehr breit aufgefasst wird. Diese wird einerseits als wissenschaftliche Disziplin verstanden, die sich mit Prozessen des Fremdsprachenlehrens und -lernens beschäftigt (synonymer Gebrauch zu dem im deutschen Sprachraum verbreiteten Terminus Sprachlehr- und -lernforschung). Andererseits umfasst Glottodidaktik auch den Prozess des Unterrichtens einer Fremdsprache (vgl. Grucza 1976; Pfeiffer 2001).
} 
untersuchte. Die Ergebnisse ihrer Studie haben gezeigt, dass die Kenntnis der Kollokationen nach dem Lesen eines Romans in der Untersuchungsgruppe um $3 \%$ im Vergleich zu der Kenntnis vor dem Lesen gestiegen ist. Demgegenüber zeigen die Ergebnisse aus der Kontrollgruppe, in der zusätzlich zum Lektürelesen auch eine Kollokationsschulung (durch kontrastiv angelegte Übungen) erfolgte, dass sich dank diesen Übungen die produktive Kollokationskompetenz der Untersuchten um $16 \%$ verbesserte. Dies zeigt, dass die Rezeption der Kollokationen nicht automatisch zu deren Beherrschung führt und dass zum produktiven Internalisieren der Kollokationen ein bestimmtes Maß an Aufmerksamkeit erforderlich ist. Zu erwähnen ist noch eine Studie von Holderbaum (2003: 78), die den Gebrauch von Kollokationen in Schulaufsätzen der Grund- und Hauptschüler im Deutsch-als Mutterspracheunterricht analysierte, wobei sie sich auf die in den Schüleraufsätzen gebrauchten Kollokationsarten konzentrierte.

Die bisher größte Studie zur Kollokationsforschung aus Sicht der DaF-Lehr- und -Lernforschung stammt von Reder (2006). Ihr Interesse galt dem Gebrauch von Kollokationen von ungarischen Lernenden. In der quantitativ ausgerichteten Studie wurden einige Gruppen, darunter DaF-Abiturienten, DaM-Abiturienten und Germanistikstudenten untersucht. Das Datenkorpus bildeten schriftliche Tests, in denen die Kenntnis der Kollokationen in Übersetzungstest mit Lückenübungen geprüft wurde. Da als Forschungsmethode die Fehleranalyse gewählt wurde, wurden für diese Untersuchung solche Kollokationen gewählt, bei denen die Interferenzen aus der L1 und aus der L2 vermutet wurden. Eines der Ergebnisse der Studie lautete: "Negativer L1-Transfer der Kollokationsbestandteile in zielsprachlichen Lerneräußerungen verhält sich quantitativ reziprok zum Sprachniveau der Lerner" (ibd.: 147), d. h. die Zahl der L1-Interferenzfehler sinkt mit dem steigenden Sprachniveau. Der geringste negative Transfer aus der L1 wurde nämlich bei Germanistikstudierenden ermittelt. Die Studie hat auch ergeben, dass mit dem fortschreitenden Niveau die Zahl der L2-bedingten Fehler steigt. Dabei konnten folgende Fehlerquellen ermittelt werden: Simplifizierung, Analogiebildung und Paraphrasierung des Kollokators. Leider ist es nicht sicher, woraus dies resultiert: aus dem fortgeschritteneren Sprachniveau der Teilnehmer, aus der Kenntnis der geprüften Kollokationen, aus der Entwicklung neuer Wortschatzlernstrategien (kollokationales Lernen) oder auch aus dem höher entwickelten Kollokationsbewusstsein. Da in der Studie sehr wenige Informationen über die Untersuchungsteilnehmer (insbesondere hinsichtlich ihres bisherigen Wortschatzlernens, ihrer Bewusstheit der Kollokationen usw.) dargestellt wurden, werfen die Ergebnisse der Untersuchung wenig Licht auf die Gründe für die erfasste Wirklichkeit, geschweige denn geben sie eine Antwort auf die Frage nach dem Prozess der Entwicklung von Kollokationskompetenz. Eigentlich ist die Publikation von Reder die einzige größere Arbeit, in der Kollokationen aus der fremdsprachendidaktischen Sicht bzw. aus der Perspektive der Fremdsprachenerwerbsforschung (in Bezug auf DaF) thematisiert worden sind.

Erwähnt werden kann an dieser Stelle noch eine Monographie von Lütge (2002), die sich zwar mit dem Lernen von Syntagmen im Fremdsprachenerwerb beschäftigte, wobei sich ihr Forschungsinteresse eigentlich auch auf Kollokationen bezog. In dieser Studie wurde untersucht, auf welche unbekannten lexikalischen Einheiten die Englisch-als-Fremdsprache-Lernenden beim Rezipieren der Lesetexte achten: auf Einzelwörter bzw. auf syntagmatische Wortverbindungen. Außerdem sollte der Frage nach der Wirksamkeit der Aufmerksamkeitslenkung für den Erwerb von Syntagmen nachgegangen werden. Aus dieser Untersuchung geht u. a. hervor, dass die Lernenden sich stärker auf unbekannte Einzelwörter konzentrierten und seltener Syntagmen als die nachzuschlagenden LE fokussieren. Die Aufmerksamkeitslenkung auf syntagmatische Wortverbindungen kann deren Erwerb begünstigen, wobei sich die explizite und implizite Aufmerksamkeitslenkung unterschiedlich auf verschiedene Lernertypen auswirkte, was folgendermaßen formuliert wurde: "Es zeigte sich ferner, dass die Aufmerksamkeitslenkung sich nicht nur positiv auf die fokussierten Items auswirkte, sondern allgemein zu einer stärker 
formorientierten Rezeption des Textes führte. [...] Individuelle Faktoren können dies zudem noch beeinflussen." (ibd.: 234)

\subsection{Bewusstheit der Kollokationen und kollokationales Wortschatzlernen unter DaF- Lernenden}

Wie oben ausgeführt, könnte die Kenntnis des Kollokationsbegriffs von dessen Relevanz in der DaF-Didaktik zeugen. Aus diesem Grunde wurde zu Anfang der Studienjahre 2012 und 2013 eine Umfrage unter den Germanistik/DaF-StudienanfängerInnen an der Ermland-MasurenUniversität in Olsztyn (Polen) durchgeführt. Zwar wurde diese Untersuchung an einer einzigen polnischen Universität durchgeführt, aber die Befragten kamen aus unterschiedlichen Orten und Schulen (aus dem Umkreis von etwa $300 \mathrm{~km}$ ) an diese Universität, sodass diese Untersuchung als eine repräsentative Studie für den nord- und nordöstlichen Teil Polens aufgefasst werden kann. ${ }^{19}$ Aus der Fragebogenerhebung zum bisherigen Wortschatzlernen und zu lexikalischen Strategien der Befragten, an der 107 DaF-Lernende teilnahmen, geht hervor, dass der Kollokationsbegriff für 84,2\% der Befragten unbekannt war. Nur 15,8\% von ihnen haben auf die Kenntnis (manchmal ungefähr, in einigen Fällen jedoch ganz genau) dieses Begriffs hingewiesen. Die untersuchten GermanistikstudiumanfängerInnen sollten auf die folgende (in ihrer Muttersprache gestellte) Frage antworten: "Ist dir der Begriff Kollokationen bekannt? Wenn ja, versuche ihn mit eigenen Worten zu erklären." Anschließend sollten die Befragten angeben, auf welche Wortarten sich Kollokationen beziehen und möglicherweise einige Beispiele für diese liefern. Von 107 Befragten haben nur 17 (15,8 \%) mit einer mehr oder weniger zufrieden stellenden Antwort erklärt, was Kollokationen sind, wodurch sich die Kenntnis dieses Begriffs belegen lässt. Die Erklärungen reichen von ganz simplen ("Kollokation ist eine Verbindung"20, "Eine Substantiv-Verb-Phrase", "Verbindung eines Substantivs mit einem Verb", "Verbindung eines Verbs mit einem entsprechenden Substantiv") über konkretere bis zu sehr gut durchdachten und gut charakterisierenden Beschreibungen des uns interessierenden Begriffs:

- "Kollokationen sind eine feste Verbindung der Wörter, die eine Phrase bilden."

- "Ein Verb fügen wir an ein passendes Substantiv hinzu."

- "Behalten eines Verbs mit einem entsprechenden Substantiv."

- "Lernen von Phrasemen."

- "Das ist eine Verbindung zweier Wörter; die Angabe des Verbs zu einem Substantiv."

- "Unterordnung einer Wortart der zweiten (z. B. den Verben ordnen wir Substantive unter)."

- $\quad$ "Einem Substantiv wird ein Verb zugeordnet, das mit der polnischen Übersetzung nicht deckungsgleich sein muss."

- "Eine entsprechende Zusammenstellung der Wörter, so dass sie eine Einheit bilden."

- "Eine Verbindung der Wörter, die oft zusammen vorkommen, und in dieser Zusammenstellung haben sie eine konkrete Bedeutung."

Aus den Erklärungen des Kollokationsbegriffs geht jedoch hervor, dass fast alle Befragten, denen der Begriff Kollokation bekannt zu sein schien, diesen nur mit der Substantiv-Verb-Verbindung assoziierten. Lediglich eine Person hat geantwortet, dass Kollokationen eine Art der

\footnotetext{
${ }^{19}$ Die Befragten kamen aus über 80 unterschiedlichen Schulen aus unterschiedlichen Orten, was zusätzlich noch für die Repräsentativität dieser Daten hinsichtlich ihrer geographischen Verteilung spricht.

${ }^{20}$ Die auf Polnisch formulierten Äußerungen der Befragten wurden von der Autorin des vorliegenden Beitrags ins Deutsche übersetzt.
} 
Verbindung von Verben und Adjektiven sein kann, aber dafür wurden von diesem Befragten folgende Beispiele angegeben (Zähne putzen, im Bett bleiben).

Eine weitere Untersuchung, die von der Autorin des vorliegenden Beitrags durchgeführt wurde, bezog sich auf die Erfassung von Wortschatzlernstrategien der Studienanfänger der Jahre 2010 bis 2012. Dabei handelte es sich um die Ermittlung des Lernens der neuen lexikalischen Einheiten in syntagmatischen Beziehungen. Da wie oben gezeigt wurde, dem überwiegenden Teil der untersuchten Germanistikstudierenden zu ihrem Studienanfang der Begriff Kollokation nicht bekannt war, wurden die Befragten danach gefragt, wie oft sie zum einen Einzelwörter und zum anderen, wie oft sie ein Substantiv mit einem dazu gehörigen Verb (syntagmatisches Wortschatzlernen) lernen. ${ }^{21}$ An dieser Untersuchung nahmen 149 Befragte teil, die ihr Germanistikstudium in den Jahren von 2010 bis 2012 aufgenommen haben. Zum Teil handelte es sich um die gleichen Probanden, die oben angesprochen wurden. Die Ergebnisse der Fragebogenerfassung zeigen, dass fast $70 \%$ der Befragten den neuen Wortschatz in Form von Einzelwörtern lernen, wobei $24,2 \%$ dies sehr oft und $44,3 \%$ oft tun. Demgegenüber lernen nur $15 \%$ der Befragten das Substantiv in einer Verbindung mit dem mit ihm zu verbindenden Verb $3,5 \%$ lernen neue LE auf diese Weise sehr oft und 11,5\% oft). 27,3\% der untersuchten Studienanfänger machen das nur manchmal, $40 \%$ machen das nur selten und 17,7 \% nie. Dies zeigt eindeutig, dass das Lernen von syntagmatischen Wortverbindungen und darunter auch von Kollokationen in dieser Befragtengruppe eine Seltenheit darstellte (vgl. Targońska 2014).

Die Ergebnisse der raumbegrenzten Untersuchung können auch als ein Hinweis auf die Vernachlässigung der Kollokationen im schulischen DaF-Unterricht interpretiert werden, ${ }^{22}$ denn die zu ihrem Studienbeginn befragten Studierenden wurden auch von über 100 DaF-Lehrkräften unterrichtet, die sie weder auf Kollokationen noch auf die Relevanz des kollokationalen Lernens hingewiesen zu haben scheinen. In der diese schriftliche Befragung ergänzenden mündlichen Befragung in Form von Interviews ${ }^{23}$ kam eindeutig zum Ausdruck, dass bei der Tafelanschrift bei der Wortschatzeinführung und Semantisierung die neuen LE (sowohl Verben als auch Substantive) von den DaF-Lehrkräften nie in ihren syntagmatischen Verbindungen präsentiert worden waren. Manche Lehrkräfte haben sogar mit dem Wortschatz nicht gezielt gearbeitet, geschweige denn diesen explizit präsentiert und eingeführt. In solchen Fällen mussten die Lernenden die neuen in dem dem Lehrwerk beigefügten Glossar aufgelisteten Vokabeln selbstständig lernen, wobei diese dort als Einzelwörter präsentiert worden sind. Dies kann ein Grund dafür sein, warum den DaF-Lernenden in größerem Maße der Kollokationsbegriff und dessen Relevanz nicht geläufig waren. Außerdem wurde das Kollokationslernen von ihnen nicht in das Repertoire der Wortschatzlernstrategien aufgenommen, was zweifelsohne nicht ohne Bedeutung für ihre lexikalische Kompetenz war.

Im Folgenden wird aus Platzgründen keine eingehende Analyse der DaF-Lehrwerke hinsichtlich der Thematisierung von Kollokationen vorgenommen. ${ }^{24}$ An dieser Stelle kann nur eine

21 Die Autorin des vorliegenden Beitrags ist sich dessen bewusst, dass bei dieser Fragestellung insbesondere das
Lernen von Substantiv-Verb-Syntagmen fokussiert wurde, wobei nicht jede solche syntagmatische Verbindung
eine Kollokation bildet. Diese Art der Syntagmen kann jedoch als ein breites Verständnis des Kollokationsbegriffs,
das für den Britischen Kontextualismus typisch war, aufgefasst werden. Im Folgenden wurde davon ausgegangen,
dass das gleichzeitige Lernen des Substantivs in der Verbindung mit einem Verb auch Kollokationslernen bedeu-
ten bzw. umfassen könnte. Dem liegt die Annahme zu Grunde, dass das Lernen der lexikalischen Einheiten in
syntagmatischen Verbindungen implizit auf das Lernen der Kollokationen hinweisen kann.
22 Es kann natürlich nicht ausgeschlossen werden, dass Kollokationen als ein sprachliches Phänomen auch im
Muttersprachenunterricht der Befragten nie behandelt wurden und die Befragten kein Kollokationsbewusstsein in
diesem Unterricht bzw. auch im Unterricht weiterer Fremdsprachen entwickelt haben.
23 Die Interviews hinsichtlich der Wortschatzlernstrategien und der schulischen Wortschatzarbeit wurden mit 26
Befragten durchgeführt.
24 Man könnte hier einwenden, dass gerade die Analyse der gängigen DaF-/DaZ-Lehrwerke als ein wichtiger
Faktor zu betrachten ist, der auf eine mögliche (Nicht)Vernachlässigung der Kollokationen verweisen könnte. Da 
einzige der Autorin des Beitrags bekannte größere Forschungsarbeit bezüglich der Lehrwerkanalyse genannt werden, in der nur nebenbei auch Kollokationen thematisiert wurden. Es handelt sich um die Arbeit von Zenderowska-Korpus (2004) ${ }^{25}$, deren Ziel die qualitative und quantitative Erfassung der "sprachlichen Schematismen" (dazu zählt die Autorin Phraseologismen, Sprichwörter, geflügelte Worte, Funktionsverbgefüge, Höflichkeits- und gesprächsspezifischen Formeln und Kollokationen) in ausgewählten Lehrwerken war. Die letzteren wurden gemeinsam mit Funktionsverbgefügen in der Gruppe der grammatisch-lexikalischen Schematismen positioniert. Zenderowska-Korpus zählte jedoch zu Kollokationen viele syntagmatische Wortverbindungen, die eigentlich keine Kollokationen im engeren Sinne darstellen (z. B. Hausaufgaben/Frühstück machen, ein Instrument spielen, die Umwelt schützen). Man kann jedoch nur auf Grund dieser Lehrwerkanalysen, die vor 10 Jahren durchgeführt wurden, noch nicht auf die Vernachlässigung der Kollokationen in den neueren Lehrwerken schließen. Es kann nämlich nicht ausgeschlossen werden, dass in den neueren Lehrwerken Kollokationen mehr Raum eingeräumt wird. Dazu wären jedoch noch weitere Untersuchungen notwendig, die für diesen Beitrag nicht durchgeführt werden konnten.

Aus der Sicht der Autorin des vorliegenden Beitrags und aufgrund ihrer Erfahrung als DaFLehrkraft kann man nur sagen, dass in der letzten Zeit immer häufiger (aber noch nicht in ausreichendem Maße) Wortschatzübungen anzutreffen sind, in denen die Verbindung von Substantiven mit Verben thematisiert wird. Allerdings handelt es sich bei diesen Substantiv-VerbVerbindungen selten um Kollokationen im engeren Sinne. Jedoch ist mir kein gängiges DaFLehrwerk (von der A1- bis zu B2-Stufe) bekannt, in dem Kollokationen als eine sprachliche Erscheinung explizit gebraucht und thematisiert worden wären. ${ }^{26}$ Dies kann auch der Grund dafür sein, warum von den meisten Lehrkräften nie Bezug auf Kollokationen genommen wird und diese nicht explizit thematisiert worden sind. Der Analyse der DaF-Lehrwerke müssten jedoch andere Forschungsarbeiten gewidmet werden.

\section{$5 \quad$ Zusammenfassung und abschließende Bemerkungen}

In dem vorliegenden Beitrag wurden viele Indizien genannt, die auf eine Vernachlässigung der Kollokationen in der DaF-Didaktik hinweisen. Dabei wurden nur einige dieser Indizien analysiert. Zusammenfassend kann festgestellt werden, dass Kollokationen im DaF-Bereich nicht explizit genug behandelt werden. Dies bezieht sich in erster Linie auf die Erklärung des Kollokationsbegriffs als auch auf die Behandlung dieses wichtigen Sprachphänomen in DaF-Nachschlagewerken. Die Analyse der DaF-Handbücher bestätigt, denn nur in einem der 15 analysierten Nachschlagewerke (vgl. Krumm et al. 2010) der Kollokationsbegriff nicht nur in einem Register bzw. Glossar und im Text zur Wortschatzarbeit bzw. zum Wortschatzlernen, sondern auch in einem separaten Unterkapitel thematisiert wird. In den meisten Handbüchern konnten einige wenige Sätze zu Kollokationen ausfindig gemacht werden, denen nur spärliche Informationen zu dieser sprachlichen Erscheinung und deren Rolle entnommen werden können. Es gibt aber auch solche Werke, in denen Kollokationen überhaupt nicht thematisiert wurden. Im Allgemeinen kann festgestellt werden, dass DaF-Handbücher Kollokationen in großem Maße vernachlässigen - indem dort weder auf die Spezifik noch auf die Relevanz dieses Sprachphänomens hingewiesen wird - wobei die Publikationen von Krumm et al. (2010) und Koeppel (2010) als Einzelphänomene und erste positive Erscheinungen gesehen werden können.

\footnotetext{
aber der Analyse von drei Lehrwerken (und dies ist aus der Sicht der Autorin des vorliegenden Beitrags die Mindestzahl der untersuchten Lehrwerke in einem wissenschaftlichen Beitrag) oft ganze Monografien, Magister- und Doktorarbeiten gewidmet werden, so könnte eine gründliche Analyse zweier Lehrwerke (die stets ein Lehrwerk mit all seinen Bänden und Komponenten umfassen sollte) den Rahmen dieses Beitrags sprengen.

${ }^{25}$ Ihre Analyse bezog sich auf drei Lehrwerke (zwei europaweit eingesetzte deutsche und ein polnisches Lehrwerk).

${ }^{26}$ Derselben Meinung ist auch Reder (2011: 138), die Folgendes feststellt: "Mir ist kein überregionales DaFLehrwerk bekannt, das Lerner mit dem Kollokationsbegriff vertraut machen würde."
} 
Obwohl Kollokationen bereits als übersetzerisches bzw. dolmetscherisches und als lexikographisches Problem erkannt wurden (wenngleich Forschungsarbeiten auch in diesen Gebieten Desiderat sind), ist das Fehlen von empirischer fremdsprachendidaktisch orientierter Kollokationsforschung in Bezug auf DaF zu verzeichnen. Festzuhalten ist außerdem die Vernachlässigung der Kollokationen im DaF-Unterricht. Man kann die These formulieren, dass die Bewusstheit der Kollokationen und der Relevanz des kollokationalen Lernens als ein vernachlässigter Faktor der Kollokationen in der DaF-Didaktik bezeichnet werden kann (vgl. Kap. 4.4). Aus den oben präsentierten Forschungsergebnissen geht nämlich hervor, dass zumindest in der untersuchten Gruppe von Studierenden, die ihr Germanistikstudium aufnehmen, Kollokationen ein unbekanntes sprachliches Phänomen darstellen. ${ }^{27}$ Sowohl dieser Begriff als auch diese spezifische Art der syntagmatischen Wortverbindung ist ihnen größtenteils nicht bekannt und die Handvoll von denjenigen, die mit dem Kollokationsbegriff etwas anfangen können, können fast als eine Ausnahme aufgefasst werden. Aus diesem Grund ist es auch nicht verwunderlich, dass die meisten befragten DaF-Lernenden bis dahin kollokationales Lernen nicht in das Repertoire der lexikalischen Strategien aufgenommen haben und nach wie vor den neuen Wortschatz als eine Ansammlung von vereinzelten Wörtern lernen. Schuld daran könnten zwar die DaF-Lehrkräfte (damit auch die DaF-Lehrerausbildung und die DaF-Lehrerfort- und -weiterbildung) oder die Lehrwerke bzw. Lehrwerkautoren sein. Aber auch der muttersprachliche Unterricht ${ }^{28}$ kann dafür verantwortlich sein, wenn dort dieses sprachliche Phänomen nicht thematisiert wird. Das Problem ist sehr komplex und deren Ursachen könnten auch in einer falschen Annahme begründet sein, dass der Spracherwerb Grammatikerwerb heißt und dass der Wortschatzerwerb mit dem Erwerb von Einzelwörtern gleichzusetzen ist, wobei jedes Einzelwort seine Bedeutung trägt (Phraseologie ist gerade der Wortschatz- bzw. Sprachbereich, in dem sichtbar ist, dass dies nicht stimmt). Auch falsches Sprachauffassen oder das Missachten der Tatsache, dass ein Großteil der Sprachverwendung formelhaft-phraseologisch ist (vgl. Stein 1995), bleibt nicht ohne Bedeutung für diese vernachlässigte Position der Kollokationen (auch innerhalb der Phraseodidaktik) in der DaF-Didaktik. In Anlehnung an die oben dargestellte Untersuchung (vgl. Kap. 4.4) kann man auch darauf schließen, dass Kollokationen und die Notwendigkeit des kollokationalen Lernens möglicherweise auch den Lehrkräften nicht bewusst sein kann.

Vernachlässigung ist auch bezüglich der fremdsprachendidaktisch orientierten Kollokationsforschung festzuhalten. Dabei handelt es sich sowohl um die Thematisierung der Kollokationen als sprachliches bzw. lexikalisches Phänomen im DaF-Unterricht als auch um Aspekte des Lernens von Kollokationen im DaF-Unterricht. Wir wissen nämlich nicht viel darüber, ob Kollokationen im europaweiten DaF-Unterricht thematisiert werden. Außerdem ist es noch nicht bekannt, wie die Kollokationskompetenz entwickelt werden kann und welche Faktoren bei deren Entwicklung bzw. Förderung eine Rolle spielen können. Unerforscht bleiben weiterhin die Entwicklung des Kollokationsbewusstseins sowie die Tatsache, ob ein impliziter Hinweis auf Kollokationen (z. B. durch Semantisierung unbekannter lexikalischen Einheiten mittels Einführung von Kollokationen bzw. durch Kollokationsübungen) zur Entwicklung des Kollokationsbewusstseins beitragen kann oder, ob ein expliziter Hinweis auf Kollokationen zur Entwicklung des Kollokationsbewusstseins bei allen Lernertypen notwendig ist. Es wäre interessant nachzuprüfen, ob das in einer Fremdsprache entwickelte Kollokationsbewusstsein auf die weitere Tertiärsprache übertragen wird, ob kollokationales Lernen als lexikalische Wortschatzlernstrategie von den Fremdsprachenlernenden von einer Fremdsprache auf die weitere gerade zu erlernende

\footnotetext{
${ }^{27}$ Da über $90 \%$ der Befragten fünf Monate nach ihrem Abitur untersucht worden waren und sie eine repräsentative Gruppe für einen Teil Polens (Umkreis von über $300 \mathrm{~km}$ ) bildeten, kann man vermuten, dass die Ergebnisse der Studie auch den Zustand an den polnischen Oberschulen widerspiegeln.

28 Vgl. dazu auch den Beitrag von Mückel (2011) zur Analyse der Sprachbücher unter dem Aspekt der Berücksichtigung der Phraseme in dem muttersprachlichen Deutschunterricht. Hallsteinsdóttir (2001) konnte in ihrer Forschungsarbeit nachweisen, dass die muttersprachliche phraseologische Kompetenz eine wichtige Rolle bei der Entwicklung der phraseologischen Kompetenz in der Fremdsprache hat.
} 
Fremdsprache übertragen wird. Diese an dieser Stelle gestellten Fragen weisen eindeutig darauf hin, dass eine fundierte empirische Kollokationsforschung noch in den Kinderschuhen steckt und vieles noch untersucht werden sollte.

Das Ziel dieses Beitrags ist es nicht, genaue Wege zur Behebung der Defizite hinsichtlich der Kollokationen aufzeigen, weil dies den Rahmen dieses Beitrags sprengen würde. Deshalb wird an dieser Stelle nur kurz auf mögliche Lösungen eingegangen. Man kann davon ausgehen, dass der für die Phraseodidaktik gültige Dreischritt, nämlich "Phraseologismen entdecken, entschlüsseln und verwenden" (Kühn 1992: 177-182, vgl. auch dazu Lüger 1997), sich auch in der Kollokationsdidaktik bewähren kann. Die DaF-Lernenden sollten also für Kollokationen als ein sprachliches Phänomen und zugleich für verschiedene Arten von Kollokationen sensibilisiert werden, was die Grundlage für das Entdecken von Kollokationen bildet. In Anschluss daran sollten die DaF-Lernenden die Bedeutung der Kollokationen entschlüsseln, was sich im Vergleich zu anderen Phrasemarten als viel einfacher erweisen kann. Lüger (1997: 101) schlägt vor, nach dem zweiten Schritt, also nach der Erschließung der Bedeutung von Phrasemen, die Festigungsphase anzuschließen, in der sowohl form- als auch bedeutungsbezogene Übungen einzusetzen wären.

In Anlehnung an Lütge (2002: 219-220) wird für die wichtigste zu ergreifende Maßnahme gehalten, das Bewusstsein der Fremdsprachenlehrkräfte für Notwendigkeit des syntagmatischen Lernens zu stärken. Dieses Bewusstsein könnte die Fremdsprachenlehrkräfte zu einer anderen Textarbeit veranlassen, bei der $u$. a. nicht nur die Inhalts- sondern auch Formorientierung wichtig wären. Die Fremdsprachenlernenden sollen auf Kollokationen und auf die Notwendigkeit ihres Auswendiglernens hingewiesen werden. Ebenso sollte auf die Entwicklung des Kollokationsbewusstseins bei den Fremdsprachenlernenden abgezielt werden, die an die formorientierte Sprachrezeption gewöhnt werden sollten. ${ }^{29}$ Von der Lehrkraft wäre also zu erwarten, Kollokationen als ein sprachliches Phänomen, das beim Wortschatzlernen zwar eine wichtige Rolle spielt, aber oft eine häufige Fehlerquelle darstellt, im DaF-Unterricht möglichst früh zu thematisieren. Schon auf der Etappe der Bedeutungsvermittlung sollten neue Wörter in ihren typischen Kollokationen präsentiert bzw. eine schon geläufige Kollokationsbasis durch andere neue Kollokatoren ergänzt werden. Dabei ist Löschmanns (1986) Forderung zu folgen, Wortschatzarbeit schon im Anfangsunterricht auf syntagmatischer Ebene zu betreiben. Neben dem Einsatz von expliziten Kollokationsübungen in der Übungs- und Automatisierungsphase, sollten die lexikalischen Einheiten auch in Form von Kollokationen bei der Wortschatzkontrolle abgefragt werden. Die Lehrkräfte sollten bei den DaF-Lernenden Kollokationsbewusstsein entwickeln, das höchstwahrscheinlich einen wichtigen Beitrag zur Entwicklung der Kollokationskompetenz leisten kann. Darüber hinaus müssen didaktische Schritte überlegt werden, anhand derer es gelingt, dass DaF-Lernende kollokationales Lernen in das Repertoire der Wortschatzlernstrategien aufnehmen. Darüber hinaus schlägt Lütge (2002: 228) vor, Fremdsprachenlernende mit forschendem Lernen bekannt zu machen, wobei neben den Korpora und Konkordanzen auch Wörterbücher eingesetzt werden könnten.

\section{Literaturverzeichnis}

Bahns, Jens (1987): "Kollokationen in englischen Wörterbüchern". Anglistik und Englischunterricht 32: 87-104.

Bahns, Jens (1994): "Die Berücksichtigung der Kollokationen in den drei großen Lernerwörterbüchern des Englischen". Fremdsprachen Lehren und Lernen 23: 84-101.

Bahns, Jens (1996): Kollokationen als lexikographisches Problem. Eine Analyse allgemeiner und spezieller Lernerwörterbücher des Englischen. Tübingen: Max Niemeyer.

\footnotetext{
${ }^{29}$ Mehr zu verschiedenen Übungstypen zur Bewusstmachung des Kollokationsphänomens durch formorientierte Sprachrezeption findet sich bei Lütge (2002: 222-224). Bei Bahns/Sibilis (1992) finden wir auch einen Hinweis darauf, wie Kollokationslernen mit dem Lektürelesen kombiniert werden kann.
} 
Bahns, Jens (1997): Kollokationen und Wortschatzarbeit im Englischunterricht. Tübingen: Narr.

Bahns, Jens/Sibilis, Ulrich (1992): "Kollokationslernen durch Lektüre". Neusprachliche Mitteilungen aus Wissenschaft und Praxis 45: 158-163.

Barkowski, Hans/Krumm, Hans-Jürgen (eds.) (2010): Fachlexikon Deutsch als Fremd- und Zweitsprache. Tübingen/Basel: Francke.

Barrios, Mario L. (1997): "Kollokationen - ein vernachlässigtes Thema in der Wortschatzarbeit". Zielsprache Deutsch 3/1997: 139-146.

Bausch, Karl-Richard/Christ, Herbert/Krumm, Hans-Jürgen (eds.) (1995): Handbuch Fremdsprachenunterricht. 3., überarbeitete und erweiterte Auflage. Tübingen/Basel: Francke.

Benson, Morton (1985): "Collocations and Idioms". In: Ilson, Robert F. (ed.): Dictionaries, Lexicography and Language Learning. Oxford etc., Pergamon: 61-68.

Benson, Morton/Benson, Evelyn/Ilson, Robert F. (1986): The BBI Combinatory Dictionary of English. A Guide to Word Combinations. Amsterdam/Philadelphia: Benjamins.

Bergenholz, Henning/Tarp, Sven (1994): "Mehrworttermini und Kollokationen in Fachwörterbüchern". In: Schaeder, Burkhard/Bergenholz, Henning (eds.): Fach-lexikographie. Fachwissen und seine Repräsentation in Wörterbüchern. Tübingen, Narr: 385-419.

Białek, Ewa (2009): Kolokacje w przekładzie. Studium rosyjsko-polskie. Lublin: Wydawnictwo UMCS.

Bohn, Rainer (1999): Probleme der Wortschatzarbeit. München: Langenscheidt.

Coseriu, Eugenio (1967/1978): "Lexikalische Solidaritäten". In: Geckeler, Horst (ed.): Strukturelle Bedeutungslehre. Darmstadt, Wissenschaftliche Buchgesellschaft: 239-253.

Decke-Cornill, Helene/Küster, Lutz (2010): Fremdsprachendidaktik. Eine Einführung. Tübingen: Narr.

Desselmann, Günther/Hellmich, Harald (eds.) (1986): Didaktik des Fremdsprachenunterrichts (Deutsch als Fremdsprache). Leipzig: VEB Enzyklopädie.

Durčo, Peter (2010): "WICOL - Deutsch-Slowakisches Kollokationswörterbuch". In: Korhonen, Jarmo et al. (eds.): Phraseologie global - areal - regional. Akten der Konferenz EUROPHRAS 2008 vom 13.-16.08.2008 in Helsinki. Tübingen, Narr: 255-260.

Ettinger, Stefan (1998): "Einige Überlegungen zur Phraseodidaktik". In: Eismann, Wolfgang (ed.): Europhras 95. Europäische Phraseologie im Vergleich. Gemeinsames Erbe und kulturelle Vielfalt. Bochum, Universitätsverlag Brockmeyer. 201-217.

Ettinger, Stefan (2002): "Phraseme im Fremdsprachenunterricht". In: Burger, Harald et al. (eds.): Phraseologie. Phraseology. Ein internationales Handbuch zur zeitgenösischer Forschung. Halbband 2. Berlin/New York, De Gruyter: 893-908.

Ettinger, Stefan (2013): "Aktiver Phrasemgebrauch und/oder passive Phrasemkenntnisse im Fremdsprachenunterricht. Einige phraseodidaktische Überlegungen". In: González Rey, Isabel (ed.): Phraseodidactic Studies on German as a Foreign Language. Phraseodidaktische Studien zu Deutsch als Fremdsprache. Hamburg, Dr. Kovač: 11-30. (= Lingua. Fremdsprachenunterricht in Forschung und Praxis 22).

Fandrych, Christian (2008): "Sprachliche Kompetenzen im ,Referenzrahmen'". In: Fandrych, Christian/Thonhauser, Ingo (eds.): Fertigkeiten - integriert oder isoliert? Wien, Praesens: 13-33.

Firth, John R. (1957): Papers in Linguistic, 1934-1951. London: Oxford University Press.

Forkl, Yves (2010): "Zur digitalen Zukunft der Kollokationslexikographie Perspektiven der Präsentation von Wissen über usuelle französische und deutsche Wortverbindungen in gedruckten und elektronischen Wörterbüchern". Dissertation. http://opus4.kobv.de/opus4fau/frontdoor/index/index/docId/1497, letzter Zugriff am 08.08.2014.

Gabryś-Biskup, Danuta (1990): "Some Remarks on Lexical Combinability. Lexical Collocations". In: Arabski, Janusz (ed.): Foreign Language Acquisition Papers. Katowice, Wydawnictwo Uniwersytetu Śląskiego: 31-45. 
Geyken, Alexander (2011): "Die dynamische Verknüpfung von Kollokationen mit Korpusbelegen und deren Repräsentation im DWDS-Wörterbuch". OPAL 2/2011: 9-20. http://pub.idsmannheim.de/laufend/opal/pdf/opal2011-2.pdf, letzter Zugriff am 14.11.2013.

Glaap, Albert-Reiner (1979): "Idioms im Englischunterricht - kontextualisiert, sachfeldbezogen, kontrastiv". Die Neueren Sprachen 78: 485-498.

Gładysz, Marek (2002): "Kollokationen als Übersetzungsproblem". In: Kalaga, Wojciech/Mielczarek, Zygmunt/Rachwał, Tadeusz (eds.): Literature and Linguistics. Literatur und Linguistik. Częstochowa, Wydawnictwo Wyższej Szkoły Lingwistycznej: 183194.

Gładysz, Marek (2003): Lexikalische Kollokationen in deutsch-polnischer Konfrontation. Frankfurt a. M. etc.: Lang.

Grauer, Christian (2009): Lesen, Verstehen und Übersetzen. Kollokationen als Handlungseinheiten der Übersetzungspraxis. Trier: Wissenschaftlicher Verlag Trier.

Grucza, Franciszek (1976): "Lingwistyczne warunkowania glottodydaktyki". In: Grucza, Franciszek (ed.): Glottodydaktyka a lingwistyka. Warszawa, Wydawnictwo Uniwersytetu Warszawskiego: 7-25.

Hallsteinsdóttir, Erla (2001): Das Verstehen idiomatischer Phraseologismen in der Fremdsprache Deutsch. Hamburg: Dr. Kovač. http://www.verlagdrkovac.de/volltexte/ 0435/1._Die_phraseologische_Kompetenz.pdf, letzter Zugriff am 01.02.2014.

Hallsteinsdóttir, Erla/Winzer-Kiontke, Britta/Laskowski, Marek (eds.) (2011): Phraseodidaktik. Phraseodidactics. (= Linguistik online 47).

Handwerker, Brigitte (2010): "Phraseologismen und Kollokationen". In: Krumm, Hans-Jürgen et al. (eds.): Deutsch als Fremd- und Zweitsprache. Ein internationales Handbuch. 1. Halbband. Berlin/New York, De Gruyter: 246-255.

Hausmann, Franz J. (1984): "Wortschatzlernen ist Kollokationslernen. Zum Lehren und Lernen französischer Wortverbindungen". Praxis des neusprachlichen Unterrichts 31: 395-406.

Hausmann, Franz J. (2003): "Kollokationen in der Fachsprache. Schwerpunkt Französisch". In: Jung, Udo O. H./Kolesnikova, Angelina (eds.): Fachsprachen und Hochschule. Frankfurt a. M. etc., Lang: 83-92. (= Bayreuther Beiträge zur Glottodidaktik 9).

Hausmann, Franz J. (2004): "Was sind eigentlich Kollokationen?" In: Steyer, Kathrin (ed.): Wortverbindungen - mehr oder weniger fest. Berlin, de Gruyter: 309-334.

Hausmann, Franz J. (2007): "Die Kollokationen im Rahmen der Phraseologie - systematische und historische Darstellung". Zeitschrift für Anglistik und Amerikanistik 55: 217-234.

Helbig, Gerhard/Henrici, Gerd/Krumm, Hans-Jürgen (eds.) (2001): Deutsch als Fremdsprache. Ein internationales Handbuch. Berlin/New York: de Gruyter.

Henrici, Gert/Riemer, Claudia (eds.) (2001): Einführung in die Didaktik des Unterrichts Deutsch als Fremdsprache mit Videobeispielen. Band I. Baltmannsweiler: Schneider Verlag Hohengehren.

Heyd, Gertraude (1991): Deutsch lehren. Grundwissen für den Unterricht in Deutsch als Fremdsprache. Frankfurt a. M.: Diesterweg.

Hill, Jimmie (1999): "Collocational Competence". English Teaching Professional: 3-7. www.freewebs.com/english1-0/competence.doc, letzter Zugriff am 08.08.2014.

Holderbaum, Anja (2003): Kollokationen als Problemgrößen der Sprachmittlung. Trier: Wissenschaftlicher Verlag Trier.

Holderbaum, Anja/Kornelius, Joachim (2001): "Kollokationen als Problemgrößen der Sprachmittlung". In: Lehr, Andrea et al. (eds.): Sprache im Alltag. Beiträge zu neuen Perspektiven in der Linguistik. Berlin/New York, De Gruyter: 533-545.

Hollós, Zita (2004): Lernerlexikographie: syntagmatisch. Konzeption für ein deutsch-ungarisches Lernerwörterbuch. Tübingen: Niemeyer.

Hollós, Zita (2008): "Kollokationen und weitere typische Mehrwortverbindungen in der ungarischen Lexikographie". Lexicographica 24: 121-133. 
Huneke, Hans-Werner/Steinig, Wolfgang (2005): Deutsch als Fremdsprache. Eine Einführung. 4. Auflage. Berlin: Erich Schmidt.

Kaufmann, Susan et al. (eds.) (2008): Fortbildung für Kursleitende Deutsch als Zweitsprache. Band 2: Didaktik. Methodik. Ismaning: Hueber.

Kielhöfer, Bernd (1994): "Wörter lernen, behalten und erinnern". Neusprachliche Mitteilungen aus Wissenschaft und Praxis 4/1994: 211-220.

Klosa, Annette/Storjohann, Petra (2011): "Neue Überlegungen und Erfahrungen zu den lexikalischen Mitspielern". In: Klosa, Annette (ed.): elexico. Erfahrungsberichte aus der lexikographischen Praxis eines Internetwörterbuchs. Studien zur Deutschen Sprache. Tübingen, Narr: 49-80.

Koeppel, Rolf (2010): Deutsch als Fremdsprache - Spracherwerblich reflektierte Unterrichtspraxis. Baltmannsweiler: Schneider Verlag Hohengehren.

Kollokationswörterbuch. Typische und gebräuchliche Wortverbindungen des Deutschen. http://colloc.germa.unibas.ch/web/suche/, letzter Zugriff am 08.08.2014.

Konecny, Christine (2010): Kollokationen. Versuch einer semantisch-begrifflichen Annäherung und Klassifizierung anhand italienischer Beispiele. München: Martin Meidenbauer.

Konecny, Christine/Autelli, Erica (eds.): Italienische Kollokationen. http://www. kollokation.at, letzter Zugriff am 08.08.2014.

Köster, Lutz/Neubauer, Fritz (2002): "Kollokationen und Kompetenzbeispiele in De Gruyter Wörterbuch Deutsch als Fremdsprache". In: Wiegand, Herbert E. (ed.): Perspektiven der pädagogischen Lexikographie des Deutschen II. Untersuchungen anhand des "de Gruyter Wörterbuchs Deutsch als Fremdsprache". Tübingen, Niemeyer: 283-310. (= Lexicographica Serien Major 110).

Kratochvílová, Iva (2004): Usuelle "Wortverbindungen. Fokussierung des aktuellen Themas "Kollokabilität lexikalischer Einheiten" auf den Bereich des deutsch-tschechischen Sprachkontrasts". Brünner Beiträge zur Germanistik und Nordistik. Sbornik praci filozofické fakulty Brněnské univerzity R 9/2004: 97-108.

Kromann, Hans-Peder (1989): "Zur funktionalen Beschreibung von Kollokationen und Phraseologismen in Übersetzungswörterbüchern". In: Gréciano, Gertrud (ed.): Europhras 88. Phraséologie Contrastive. Actes du Colloque International Klingenthal - Strasbourg. Strasbourg, Universtité dees Sciences Humaines, Département d'Etudes Allemandes: 265-271.

Krumm, Hans-Jürgen et al. (eds.) (2010): Deutsch als Fremd- und Zweitsprache. Ein internationales Handbuch. 1. Halbband. Berlin: De Gruyter.

Kühn, Peter (1992): "Phraseodidaktik. Entwicklungen, Probleme und Überlegungen für den Muttersprachenunterricht und den Unterricht Deutsch als Fremdsprache". Fremdsprachen Lehren und Lernen 21: 167-189.

Kühn, Peter (2010): "Wörterbücher/Lernerwörterbücher". In: Krumm, Hans-Jürgen et al. (eds.): Deutsch als Fremd-und Zweitsprache. Ein internationales Handbuch. 1. Halbband. Berlin, De Gruyter: 304-315.

Larreta Zulategui, Juan P. (2006): "Zu einer Klassifikation der verbalen Kollokationen". Deutsch als Fremdsprache 1/2006: 22-26.

Leśniewska Justyna (2008): "Słowniki języka angielskiego a kolokacje". In: Jodłowiec, Maria/Niżegorodcew, Anna (eds.): W stronę nowoczesnego nauczania języków obcych. Kraków, Tertium: 193-202. (= Język a komunikacja 22).

Löschmann, Martin (1986): "Die Arbeit an lexikalischen Kenntnissen". In: Desselmann, Günter/Hellmich, Harald (eds.): Didaktik des Fremdsprachenunterrichts (Deutsch als Fremdsprache). Leipzig, Enzyklopädie: 141-166.

Löschmann, Martin (1993): Effiziente Wortschatzarbeit. Alte und neue Wege. Frankfurt a. M. etc.: Lang.

Lüger, Heinz-Helmut (1997): "Anregungen zur Phraseodidaktik". Beiträge zur Fremdsprachenvermittlung 32: 69-120. 
Lütge, Christiane (2000): "Sprachbewusstheit und Wortschatzerwerb - zu Kollokationskompetenz fortgeschrittener Lerner des Englischen". In: Aguado, Karin/Hu, Adelheid (eds.): Mehrsprachigkeit und Mehrkulturalität. Dokumentation des 18. Kongresses für Fremdsprachendidaktik, veranstaltet von der Deutschen Gesellschaft für Fremdsprachenforschung (DGFF) Dortmund, 4.-6. Oktober 1999. Berlin, PVZ: 343-349.

Lütge, Christiane (2002): Syntagmen und Fremdsprachenerwerb. (Ein Lernersprachenproblem). Frankfurt a. M. etc.: Lang.

Marton, Waldemar (1977): "Foreign Vocabulary Learning as Problem No. 1 of Language Teaching at the Advanced Level". The Interlanguage Studies Bulletin 2: 33-57.

Meerholz-Härle, Birgit (2008): "Wortschatzvermittlung im DaZ-Unterricht". In: Kaufmann, Susan et al. (eds.): Fortbildung für Kursleitende Deutsch als Zweitsprache. Band 2: Didaktik. Methodik. Ismaning, Hueber: 1-34.

Möhring, Jupp (2011): "Kollokationen im Lernerwörterbuch - Anspruch und Wirklichkeit". Linguistik online 47: 33-53.

Mückel, Wenke (2013): "Phraseme im muttersprachlichen Deutschunterricht. Eine exemplarische Untersuchung von Sprachbüchern der Sekundarstufe I". In: Konecny, Christine/Hallsteinsdótir, Erla/Kacjan, Brigita (eds.): Phraselologie im Sprachunterricht und in der Sprachdidaktik. Phraseology in language teaching and in language didactics. Bielsko-Bia etc., Zora: 19-43.

Müller, Thomas (2011): Aware of Collocations. Ein Unterrichtskonzept zum Erwerb von Kollokationskompetenz für fortgeschrittene Lerner des Englischen. Frankfurt a. M. etc.: Lang.

Neveling, Christiane (2004): Wörterlernen mit Wörternetzen. Eine Untersuchung zu Wörternetzen als Lernstrategie und als Forschungsverfahren. Tübingen: Narr.

Pfeiffer, Waldemar (2001): Nauka języków obcych. Od praktyki dla praktyki. Poznań: Wagros. Porzig, Walter (1934/1973): "Wesenhafte Bedeutungsbeziehungen". In: Schmidt, Lothar (ed.): Wortfeldforschung. Zur Geschichte und Theorie des sprachlichen Feldes. Darmstadt, Wissenschaftliche Buchgesellschaft: 78-103.

Pöll, Bernhard (1997): "Syntagmatisches Wortschatzlernen. Überlegungen zu Kollokationen in Lehrbüchern und (Lerner-) Wörterbüchern des Portugiesischen". In: Stegu Martin/de Cillia, Rudolf (eds.): Fremdsprachendidaktik und Übersetzungswissenschaft. Frankfurt a. M. etc., Lang: $325-343$.

Quasthoff, Uwe (2011): Wörterbuch der Kollokationen im Deutschen. Berlin/New York: De Gruyter.

Reder, Anna (2001) "Ein heißer Tipp - oder Kollokationslernen durch eine Ganzschrift". In: Häcki Buhofer, Annelies/Durco, Peter (eds.): Wortschatz. Aneignung und Unterricht. IDT Publikation 2001. https://sites.google.com/site/kollokator/home/aufsaetze, letzter Zugriff am 03. September 2014.

Reder Anna (2002): "Eine Aufgaben- und Übungstypologie zur Entwicklung der Kollokationskompetenz von DaF-Lernern". Jahrbuch der ungarischen Germanistik: 293-311.

Reder, Anna (2006): Kollokationen in der Wortschatzarbeit. Wien: Praesens.

Reder, Anna (2006a): "Zum Transferverhalten ungarischer DaF-Lerner in Strukturabhängigkeit von Kollokationen". In: Karnowski, Pawel/Szigeti, Imre (eds.): Sprache und Sprachverarbeitung. Language and Language-Processing. Frankfurt a. M. etc., Lang: 337-379.

Reder Anna (2006b): "Kollokationsforschung und Kollokationsdidaktik". Linguistik online 28: $157-176$.

Reder, Anna (2011): "Kommen Kollokationen in Mode? Kollokationskonzepte und ihre mögliche Umsetzung in der Didaktik". Linguistik online 47: 131-140.

Roche, Jörg (2008): Fremdsprachenerwerb, Fremdsprachendidaktik. Tübingen: Narr.

Rössler, Andrea (2010): "Kollokationskompetenz fördern im Fremdsprachenunterricht - Ein Plädoyer". Die Neueren Sprachen 1/2010: 54-66. 
Scherfer, Peter (2008): "Lexikalische Kollokationen". In: Kolboom, Ingo/Kotschi, Thomas/Reichel, Edward (eds.): Handbuch Französisch. 2. Aufl. Berlin, Erich Schmidt: 238-245.

Schneider, Edgar, W. (1988): Variabilität, Polysemie und Unschärfe der Wortbedeutung. Band 1. Theoretische und methodische Grundlagen. Tübingen: Niemeyer.

Siepmann, Dirk (2003): "Eigenschaften und Formen lexikalischer Kollokationen. Wider ein zu enges Verständnis". Zeitschrift für französische Sprache und Literatur 112/3: 240-263.

Sinclair, John M. (1991): Corpus, Concordance, Collocation. Oxford: Oxford University Press. Stein, Stephan (1995): Formelhafte Sprache. Untersuchungen zu ihren pragmatischen und kognitiven Funktionen im gegenwärtigen Deutsch. Frankfurt a. M. etc.: Lang.

Steyer, Kathrin (2008): "Kollokationen in deutschen Wörterbüchern und in der deutschen Wörterbuchforschung". Lexicographica 24: 185-207.

Storch, Günther (1999): Deutsch als Fremdsprache - Eine Didaktik. München: Fink.

Storjohann, Petra (2011): Kollokationen. Tübingen: Narr.

Stubbs, Michael (1997): "'Eine Sprache idiomatisch sprechen'. Computer, Korpora, Kommunikative Kompetenz und Kultur". In: Mattheier, Klaus J. (ed.): Norm und Variation. Frankfurt a. M. etc., Lang: 151-167.

Surkamp, Carola (ed.) (2010): Metzler Lexikon Fremdsprachendidaktik. Ansätze - Methoden Grundbegriffe. Stuttgart/Weimar: Metzler.

Targońska, Joanna (2014): "Lexikalische Strategien der Germanistikstudierenden zu ihrem Studienbeginn". Lingwistyka Stosowana 9: 171-200.

Targońska, Joanna/Stork, Antje (2013): "Vorschläge für ein neues Modell zur Beschreibung und Analyse lexikalischer Kompetenz". Zeitschrift für Fremdsprachenforschung 24: 71108.

Tomas, Adam (2008): Kollokationen im Deutschen. Ein Versuch einer Determinierung. Saarbrücken: Müller.

Tschirner, Erwin (2010): "Wortschatz". In: Krumm, Hans-Jürgen et al. (eds.): Deutsch als Fremd- und Zweitsprache. Ein internationales Handbuch. 1. Halbband. Berlin, De Gruyter: 236-245.

Teubert Wolfgang (2004): "Feste Wortverbindungen in ein- und mehrsprachiger Sicht". In: Czicza, Daniel et al. (eds.): Wertigkeiten. Geschichte und Kontraste. Festschrift für Peter Bassola, zum 60. Geburtstag. Szeged, Grimm: 521-547.

Wanzeck, Christiane (2010): Lexikologie. Göttingen: Vandenhoeck \& Ruprecht.

Weller, Franz-Rudolf (1979): "'Idiomatizität' als didaktisches Problem des Fremdsprachenunterrichts - erläutert am Beispiel des Französischen. Die Neueren Sprachen 78: 530-554.

Wortschatz. Universität Leipzig. http://wortschatz.uni-leipzig.de/, letzter Zugriff am 08.08.2014.

Wotjak, Gerd (2007): "Überlegungen zum syntagmatisch-kombinatorischen Potenzial lexikalischer Einheiten". In: Lenk, Hartmut E. K./Maik, Walter (eds.): Wahlverwandtschaften Valenzen - Verben - Varietäten. Hildesheim, Georg Olms: 165-181. (= Germanistische Linguistik 188-189).

Wotjak, Barbara/Heine, Antje (2005): "Zur Abgrenzung und Beschreibung verbnominaler Wortverbindungen (Wortidiome, Funktionsverbgefüge, Kollokationen)." Deutsch als Fremdsprache 3/2005: 143-153.

Zenderowska-Korpus, Grażyna (2004): Sprachliche Schematismen des Deutschen und ihre Vermittlung im Unterricht DaF. Frankfurt a. M. etc.: Lang.

Zimmermann, Małgorzata (1981): "Zum Begriff der Kollokation in der Sprachwissenschaft und Glottodidaktik". Glottodidactica 14: 61-68.

Zöfgen, Ekkehard (2001): "Lexikalische Zweierverbindungen. 'Vertraute Unbekannte' im mentalen Lexikon germanophoner Französischlerner". In: Aguado, Karin/Riemer, Claudia 
(eds.): Wege und Ziele. Zur Theorie und Praxis des Deutschen als Fremdsprache (und anderer Sprachen). Baltmannsweilter, Schneider Hohengehren: 267-286.

Zöfgen, Ekkehard (2008): "Kollokationslexikografie im Aufwind. Der Dictionnaire des combinaisons de mots auf dem Prüfstand". Zeitschrift für Französische Sprache und Literatur 118: 107-124.

Żmudzki, Jerzy (2000): "Disparität von Attributierungen in polnischen und deutschen AdjektivSubstantiv-Kollokationen als Translationsproblem". Glottodidactica 27: 107-119. 